\title{
Méthodes de prévision des dégradations des structures bâties en zone d'affaissement minier
}

O. DECK

M. AL. HEIB

Y. GUENIFFEY

F. WOJTKOWIAK

LAEGO, INERIS

École des mines de Nancy

Parc de Saurupt

54042 Nancy Cedex deck@mines.impl-nancy.fr heib@mines.impl-nancy.fr gueniffey@mines. impl-nancy.fr

francis.wojtkowiak@mines. impl-nancy.fr

\section{F. HOMAND}

LAEGO

École nationale supérieure de géologie BP 40

54500 Vandceuvrelès-Nancy

Francoise.Homand@ensg. inpl-nancy.fr.

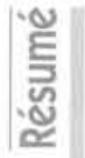

\begin{abstract}
Les mines et carrières souterraines, en activité ou abandonnées, sont susceptibles d'être à l'origine d'affaissements des terrains en surface. Qu'ils résultent de la méthode d'exploitation ou qu'ils s'avèrent accidentels, ces derniers induisent d'importants mouvements permanents en surface qui peuvent entrainer des dégradations du bâti et des infrastructures. La gestion des zones urbanisées concernées passe par la hiérarchisation du risque de dégradation et la proposition de dispositions constructives, préventives et curatives. Cet article s'intéresse à diverses méthodes de prévision des dégradations du bâti, proposées dans différents pays, qui intègrent des paramètres propres aux terrains de recouvrement, propres à l'affaissement et aux structures. Simples ou élaborées, ces méthodes sont comparées entre elles et confrontées à la réalité. Une étude statistique a été menée sur les affaissements miniers qui se sont produits dans le bassin ferrifêre lorrain dans la dernière décennie. Regroupant près de 400 individus (constructions), elle met en évidence l'existence de corrélations importantes entre les dégradations et certaines variables caractéristiques de l'affaissement ou de la structure. Il est alors possible d'élaborer une méthode de hiérarchisation du risque de dégradation, propre au bassin ferrifère lorrain.
\end{abstract}

Mots-clés : affaissements, mines, structures, interaction, dégradations, prévisions, statistique.

\section{Methods for buildings damage estimation located in mining subsidence area}

Mines and underground quarries, exploited or abandoned, can lead to ground subsidence. Whatever their origin, accident or well-known consequences of actual exploitation, they induce important permanent displacements of the ground surface which can damage buildings. Management of urbanised areas which are concerned, deals with damage criteria and remedying propositions. This paper is about different kinds of methods for building damage prevision from different countries and with different criteria related to the ground, the subsidence and structures. Theses methods are compared with one another and with reality. A statistical study was performed with mining subsidence which occurred in iron mining basin in Lorraine (France) for 1996 to 1999 . With almost 400 observations, it shows high correlations between damage level and some characteristic parameters of subsidence or structures. It is then possible to elaborate a method for damage level and risk estimation useful for the iron mining basin in Lorraine.

Key words : subsidence, mines, structures, interaction, damage, estimation, statistic. 


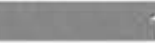 \\ Introduction}

Cette étude trouve son origine dans les affaissements miniers récents qui ont affecté le bassin ferrifère lorrain. D'une superficie de plusieurs dizaines d'hectares chacun, ces affaissements ont considérablement affecté les communes touchées. Si aucune victime corporelle n'a été déplorée, d'importants dégâts matériels ont été constatés ainsi que des conséquences psychologiques parmi les sinistrés. Les analyses qui ont fait suite à ces affaissements avaient parmi leurs objectifs, celui de se prononcer sur la vulnérabilité du bâti préexistant sur des terrains sous-minés et donc potentiellement affaissables. Cet objectif renvoie à un certain nombre de questions : dans quelle mesure les déplacements du terrain, illustrés sur la figure 1, se transmettent-ils à la structure? Quelles sont les caractéristiques du terrain, du bâti et de l'affaissement à prendre en compte pour hiérarchiser le risque? Est-il envisageable d'élaborer une méthode simple, mais efficace, pour effectuer cette hiérarchisation?

Ces questions ne sont pas nouvelles. Tous les pays miniers ont eu, à un moment de leur histoire, à s'intéresser aux conséquences en surface de leurs exploitations souterraines. Le meilleur exemple vient de l'exploitation du charbon. Pour satisfaire à la demande de nombreux gisements ont été exploités sous des zones urbanisées. Par ailleurs, des méthodes d'exploitation totale, comme celle par foudroyage, ont permis de laisser en place le minimum de matière. Un affaissement se produit alors en surface sensiblement à l'aplomb du front de taille et progresse au même rythme que l'avancement des travaux souterrains (Piguet et Wojtkowiak, 2001). Ceci explique pourquoi on trouve beaucoup d'études concernant l'impact de l'exploitation du charbon en zones urbanisées (Afrique du Sud, Angleterre, États-Unis, France, Pologne, exUnion soviétique...). L'impact des exploitations souterraines d'autres substances (calcaire, gypse, sel, fer, cuivre...) n'est guère différent si l'exploitation est profonde. Malgré des techniques d'exploitation laissant généralement en place une quantité de minerai servant de piliers pour assurer la stabilité du toit, il arrive que la charqe limite de ces derniers soit dépassée et qu'une rupture locale ou qénéralisée se produise. Mais à la différence des mines de charbon exploitées par foudroyage, l'affaissement qui se produit est accidentel et donc a priori imprévu dans le temps et dans l'espace c'est le cas des mines de fer de Lorraine. Cependant, le phénomène est comparable du point de vue des mouvements en surface au voisinage d'une structure. L'état final du mouvement peut, dans tous les cas, ètre décomposé selon deux mouvements de corps rigides (translation et rotation) et deux déformations (déformation horizontale et courbure). Les mouvements rigides du terrain se transmettront intégralement à l'ouvrage alors que les déformations pourront être gênées par la présence de l'ouvrage si ce dernier est plus rigide que le terrain. C'est pourquoi il faut distinguer la déformation horizontale et la courbure du terrain de celles de la structure. La figure 2, présentée par Geddes (1984), reprend cette décomposition en quatre mouvements élémentaires dans le cas d'une description plane du phénomène.

En caractérisant l'affaissement minier par la valeur de ces quatre mouvements élémentaires, on sous-

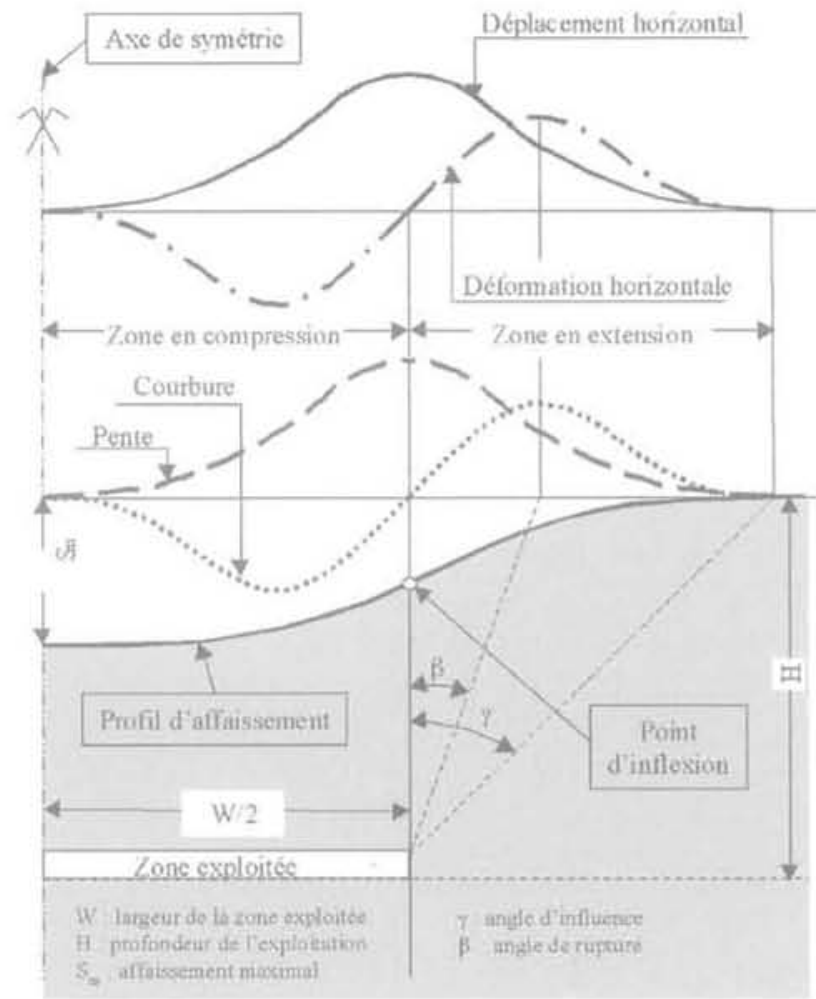

FIG: 1

Description des mouvements du terrain en surface induits par un affaissement minier. Description of ground movements occurring during mining subsidence.

entend que les conséquences d'un affaissement minier sur le bâti ne dépendent pas fondamentalement de la nature des travaux souterrains. Cette dernière influence évidemment l'amplitude des mouvements en surface mais on suppose que, pour un même mouvement généré par deux types d'exploitation différents, les conséquences sur le bâti seront les mêmes. Seule une différence de lithologie des terrains superficiels pourrait être à l'origine d'un comportement différent de l'interaction sol-structure.

Sous ces hypothèses et considérations, nous avons étudié l'impact des affaissements miniers sur le bâti lorrain. Pour ce faire, nous avons exploité une base de données recensant toutes les structures dégradées en Lorraine accompagnées d'un certain nombre de paramètres pouvant servir de critères de hiérarchisation des dégradations observées. Afin d'élaborer cette base de données et pour avoir un regard critique sur nos résultats, nous avons en premier lieu répertorié les diverses méthodes de prévision des dommages aux constructions existantes. Certains de leurs auteurs ont cherché à discriminer les dégradations au moyen d'un nombre réduit de paramètres : longueur de la structure et déformation du terrain, ou encore longueur de la structure et pente... D'autres se sont attachés à intégrer un plus grand nombre de critères au moyen de méthodes dites « des points », dans lesquelles, la résistance d'une structure est estimée grấce au nombre cumulé de points qu'on lui a attribués selon chaque critère. 


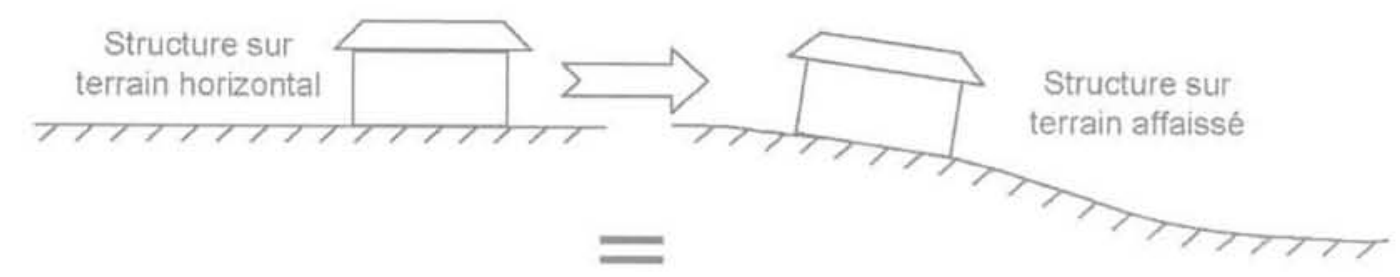

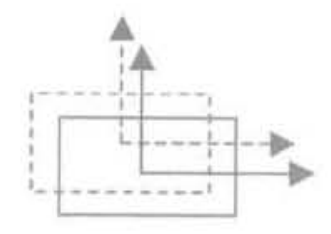

TRANSLATION

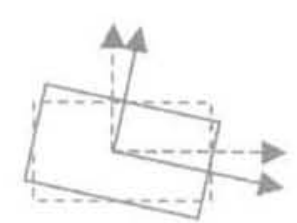

INCLINAISON

Mouvement de corps rigide

SOLLICTIATIONS ELEMENTAIRES A L'ORIGINE DES DEGRADATIONS

\section{2}

\section{Différentes démarches d'estimation des dégradations}

\section{1}

\section{Abaques à deux paramètres}

\section{917}

\section{Longueur de l'ouvrage et déformation horizontale}

La plus ancienne méthode, présentée par le National Coal Board (NCB, 1975), en Angleterre, permet une prévision des dégradations basée sur la longueur de la structure et la déformation horizontale. Elle a longtemps fait référence à travers le monde, au vu des très nombreuses publications qui en font état. Basée sur la compilation de très nombreux cas de dégradations, elle débouche sur l'abaque de la figure 3. Il a été présenté comme " un guide général pour la prédiction de l'intensité des dégradations ») qui doit être accompagné « d'une expertise difficilement réductible à des grandeurs quantifiables et dont on ne peut s'acquitter que par une grande connaissance des structures de différentes époques et différents modes de construction i). Geddes et Kennedy (1984) critiquent dans cet abaque la confusion qui est faite entre la déformation horizontale du terrain et celle de la structure, ce qui est assez courant par ailleurs. D'une part, des glissements peuvent avoir lieu à l'interface sol-structure, d'autre part, la déformation horizontale du terrain n'est pas constante le long d'ouvrages très longs $(100 \mathrm{~m}$ par exemple) puisque leur longueur est du même ordre de grandeur que les dimensions de la cuvette. Ces auteurs laissent cependant entendre que l'abaque conserve toute son utilité à deux conditions : considérer la déformation moyenne du terrain le long de l'ouvrage et supposer que la résistance de la structure est très faible par rapport aux efforts maximaux générés à l'interface solstructure, afin que la déformation des terrains soit assimilable à celle de la structure.

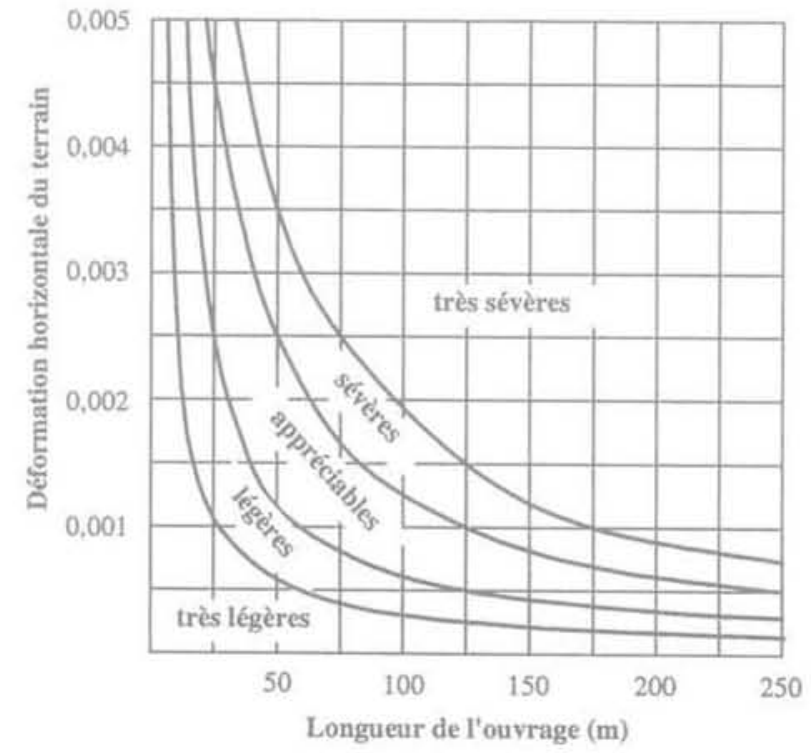

516.3 Abaque de prévision des dégradations du bâti en fonction de la longueur de l'ouvrage et de la déformation horizontale du terrain, National Coal Board (NCB, 1975).

Abacus to predict building damage in relation to horizontal ground strain and building length. National Coal Board (NCB, 1975). 
Pour nous, cet abaque est absolument inutilisable pour les ouvrages les plus courants tels que les maisons individuelles, d'une vingtaine de mètres de longueur, car il est trop imprécis. Cependant, on trouve sur la figure 4, un abaque dans le même système d'axes que celui du NCB et qui aurait été élaboré à partir de dégradations observées principalement en Afrique du Sud (Wagner et Shümann, 1991). Mais cet abaque se révèle en fin de compte n'être qu'une copie conforme de celui du NCB pour des longueurs de structure inférieures à $50 \mathrm{~m}$.

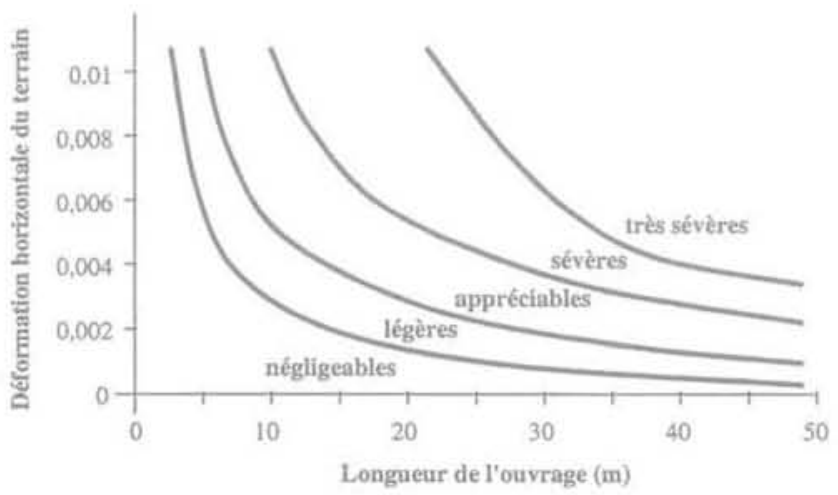

FG. 4 Abaque de prévision des dégradations du bâti en fonction de la longueur de l'ouvrage et de la déformation horizontale du terrain (Wagner et Shümann, 1991).

Abacus to predict building damage in relation to horizontal ground strain and building length (Wagner and Shümann, 1991).

\section{Q.120}

\section{Angle de distorsion et déformation horizontale}

Cet abaque théorique a été proposé par Boscardin et Cording (1989). Il est représenté sur la figure 5. Son élaboration consiste à calculer analytiquement la déformation de traction maximale $"\left(\varepsilon_{\text {structure }}\right)$ qui apparait dans une structure en maçonnerie sollicitée par la courbure du terrain " $\mathrm{R}_{\text {ternin }}$ " et par la déformation horizontale de traction uniforme du sol d'assise " $\varepsilon_{\text {termin }}$ ». Les hypothèses retenues pour ce calcul sont les suivantes : - la structure est modélisée par une poutre épaisse de longueur ( $\mathrm{L}$ » et hauteur « $\mathrm{H}$ ), reposant sur deux appuis simples, élastique, homogène et isotrope de modules élastiques « $E$ » et $\alpha G »$;

- la déformation horizontale de traction est imposée à l'ensemble de la poutre ;

- l'effet de la courbure est modélisé par l'intermédiaire d'un effort ponctuel vertical appliqué à mi-portée de la poutre afin de lui imposer une flèche équivalente à la courbure du terrain. Par ailleurs, l'axe neutre est imposé en fibre inférieure de la poutre afin de simuler l'effet rigidifiant du terrain.

Une première relation peut être établie, du type $\varepsilon_{\text {str }}$ $=f_{1}\left(L, H, E, G, R_{\text {terrain }}\right)$. Grâce aux hypothèses précédentes, l'angle de distorsion de la structure $\alpha \beta)$ (Fig. 6), généralement estimé par $\beta=L /\left(2 \cdot R_{\text {termain }}\right)$, peut être, ici, très précisément calculé par une relation du type $\beta$ $=f_{2}\left(L, H, E, G, R_{\text {temrin }}\right)$. On obtient alors finalement une relation mathématique reliant les paramètres $\varepsilon_{\text {terman' }}, \beta$ et $\varepsilon_{\text {structure }}$. En se fixant, d'une part, des propriétes méca- niques et géométriques de la poutre et, d'autre part, des valeurs seuils de $" \varepsilon_{\text {structure }}$ " qui correspondent à différents degrés de dégradation de la maçonnerie (tableau I), il est possible de construire des courbes d'iso-dégradation en fonction de la déformation horizontale du terrain et de l'angle de distorsion de la structure.

L'approche proposée est cohérente avec celle du National Coal Board (1975). Elle revient en fait à rajouter un troísième axe à l'abaque du NCB, celui de l'angle de distorsion. On peut donc construire des surfaces d'iso-dégradation plutôt que des courbes. L'abaque de Boscardin et Cording correspond d'ailleurs à la description de ces surfaces pour une longueur fixe de la structure d'environ 30 à $40 \mathrm{~m}$. Les critiques qui peuvent ètre faites sur cet abaque résident, d'une part, dans la confusion faite entre la déformation ou la courbure du sol et celles de la structure, d'autre part, dans la quantité importante d'hypothèses restrictives. Enfin, cet abaque a étê explicitement élaboré pour la zone de traction de la cuvette alors que les dégradations en zone de compression peuvent être aussi importantes, comme le montrera la suite de cet article.

\section{Sats}

\section{Pente et déformation horizontale}

L'abaque de Wagner et Shümann (1991) met en évidence des courbes d'iso-dégradation dépendantes de la déformation horizontale et de la pente du terrain (Fig. 7). La pente est, en réalité, une valeur moyenne estimée le long de la structure et qui correspond à son inclinaison (Fig. 6). Cet abaque est issu directement d'observations effectuées dans différents pays miniers (Afrique du Sud en particulier). Cependant, cette représentation est critiquable. D'une part, on observe que les individus projetés sur la figure 7 sont loin de légitimer le choix du tracé des zones d'iso-dégradation. D'autre part, cet abaque fait l'amalgame de structures de longueurs très variées. La preuve en est qu'il n'est pas compatible avec l'abaque des mêmes auteurs présenté sur la figure 4 . En effet, quelles que soient la pente et la longueur fixes d'un ouvrage, les seuils entre les zones de dégradation sont différents selon les deux abaques.

\section{2}

\section{Les méthodes multicritères}

Ces méthodes ont l'avantage, par rapport aux abaques précédents, de prendre en compte des caractéristiques techniques de structures en plus des mouvements du terrain. Elles consistent globalement à qualifier la résistance d'une structure à partir d'une description architecturale et technique. Cette qualification se fait souvent au moyen de points attribués pour chaque caractéristique, puis totalisés. Les mouvements maximaux admissibles du terrain sont alors estimés selon la classe de résistance obtenue.

\section{9:4}

\section{Les méthodes américaines}

Deux méthodes multicritères au moins ont vu le jour ces vingt dernières années. 


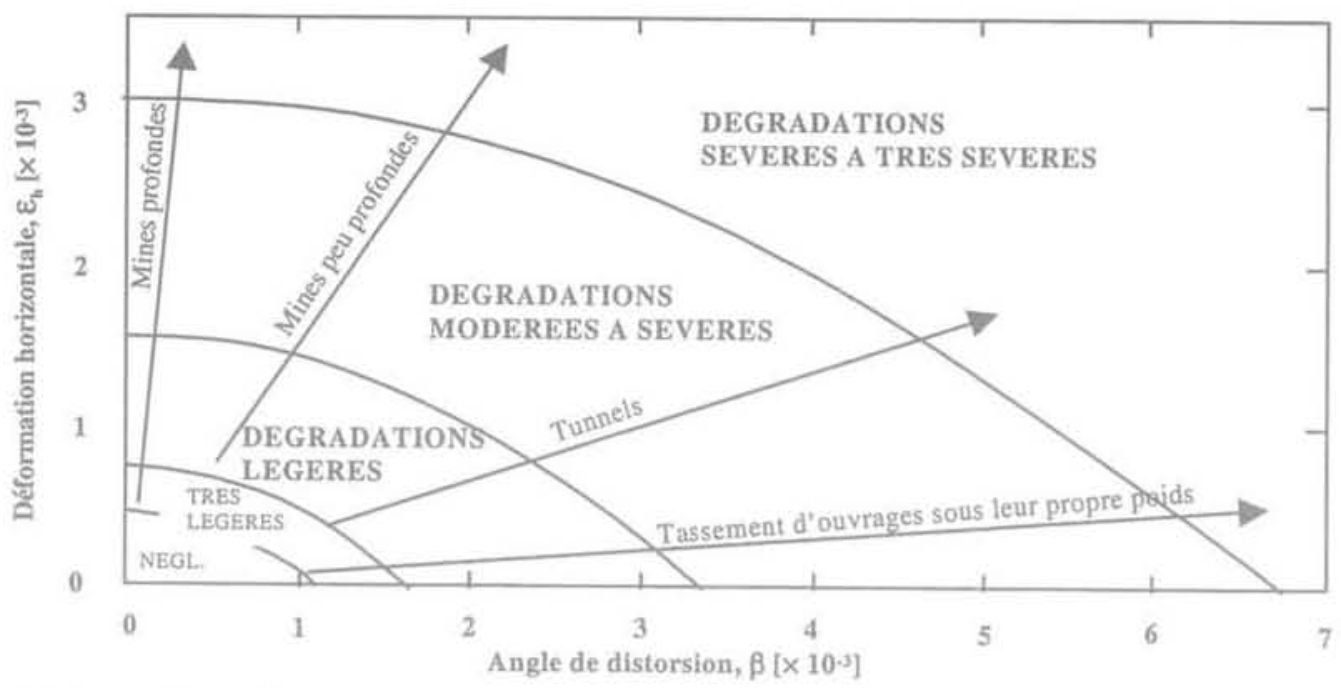

FG.5 Abaque de prévision des dégradations du bâti en fonction de l'angle de distorsion de la structure et de la déformation horizontale du terrain (Boscardin et Cording, 1989).

Abacus to predict butilding damage in relation to horizontal ground strain and building distorsion (Boscardin et Cording, 1989)

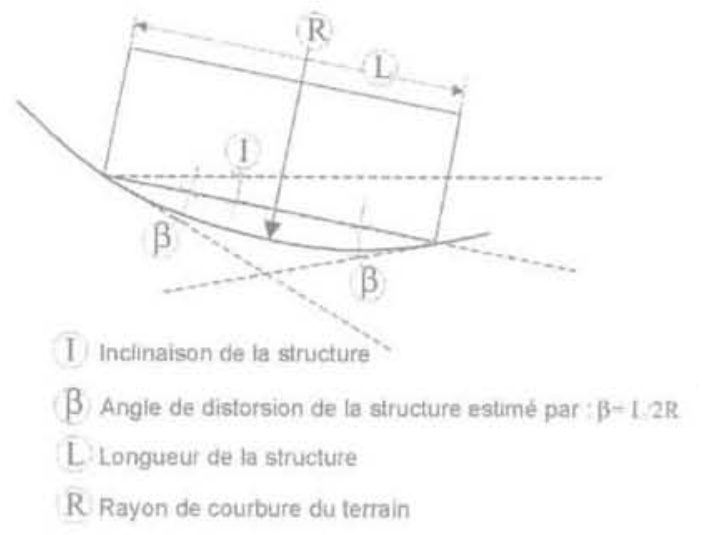

FG.6 Définition de l'angle de distorsion et de l'inclinaison d'une structure (courbure très exagérée sur le dessin).

Definition of cistorsion angle and tilt of building (exaggerate curvature on the figure).

La première méthode, proposée par Bhattacharya et Singh (1984), distingue très simplement quatre catégories de structures, selon leur système constructif. Les auteurs définissent ensuite trois degrés de dégradation auxquels ils associent des seuils de mouvement pour le terrain ou la structure selon la catégorie de l'ouvrage considéré (tableau II). Les valeurs adoptées pour les mouvements admissibles sont issues d'une synthèse bibliographique des seuils généralement admis à travers le monde. Tout en revêtant de ce fait un caractère " universel », cette démarche présente l'inconvénient de ne pas maîtriser l'origine des valeurs utilisées. Le risque d'accumulation artificielle de valeurs suite à une auto-justification des auteurs les uns par rapport aux autres n'est donc pas à exclure.

La seconde méthode, décrite par Yu et al. (1988), est plus précise sur la caractérisation des structures puisqu'elle prend en compte le type de fondation, le type de superstructure, la taille relative de l'ouvrage par rapport à celle de la cuvette ainsi que l'élancement de l'ouvrage (tableau III). L'inconvénient notable présenté par cette méthode est l'absence d'explication scientifique quant à la distribution des points selon chaque critère ou pour la définition des seuils qui diffèrent légèrement de ceux de Bhattacharya et Singh.

\section{2agas \\ Les méthodes polonaises}

Ces méthodes sont, à notre avis, les plus élaborées et probablement les mieux ajustées, Les échanges et les contacts instaurés avec l'Institut central minier de Katowice (GIG) en Pologne, ont permis de constater que leur méthode était très largement acceptée et jugée satisfaisante par toutes les parties concernées Jors d'un

TABLEAU Valeurs et seuils adoptés pour l'élaboration de l'abaque de Boscardin et Cording (1989). Values and thresholds used for the abacus of Boscardin and Cording (1989),

Caractéristiques mécaniques Coefficient de Poisson $=0,03$

Caractéristiques géométriques Hauteur et longueur de la structure identiques

Valeurs seuil de $\varepsilon_{\text {smuture }}\left(\times 10^{-3}\right)<0,5$ pour des dégradations négligeables

0,5 à 0,75 pour des dẻgradations très légères

0,75 à 1,5 pour des dègradations légères

1,5 à 3 pour des dégradations modérées à sévères

$>3$ pour des dégradations très sévères 


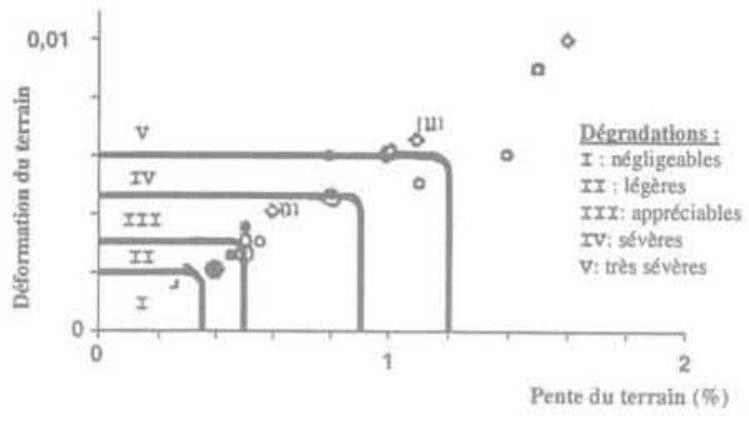

Fig.7 Abaque de prévision des dégradations du bâti en fonction de la pente du terrain et de la déformation horizontale du terrain (Wagner et Shümann, 1991).

Abacus for building damage prediction in relation to horizontal ground strain and tilt of building (Wagner and Shumann, 1991). affaissement minier, y compris les populations sinistrées. Une autre méthode émane de l'Université des mines et métallurgie de Cracovie. Elle est présentée dans le tableau IV et décrite par Dzegniuk et Hejmanowski (2000). Sept critères sont utilisés dont trois pour décrire la géométrie de la structure, trois autres pour caractériser son état technique, sa rigidité et ses renforcements et un paramètre décrivant le terrain d'assise. L'inconvénient majeur de cette méthode est de n'avoir aucune nuance sur le degré de dégradation de la structure.
La méthode élaborée à l'Institut central minier de Katowice (Kwiatek, 1998), présentée dans le tableau V. est la plus précise mais elle ne s'applique qu'aux ouvrages en maçonnerie (briques, moellons, parpaings...). La première version, fondée sur des études statistiques, date des années 1950, puis de nombreux ajustements empiriques y ont été apportés. Les utilisateurs actuels estiment qu'un taux de conformité des prévisions par rapport aux dégradations réellement observées de $85 \%$ est possible à condition d'appliquer cette méthode à un groupe de structures et non à un ouvrage isolé. Son premier inconvénient est qu'en étant exclusivement ajustée aux affaissements et structures locales, elle perd tout caractère universel. Le second reproche est, d'une part, qu'elle ne fait pas de nuance sur le degré de dégradation et, d'autre part, que le seuil de dégradation n'est défini qu'à partir d'une seule caractéristique décrivant le mouvement du terrain : la déformation horizontale.

\section{3}

\section{Une étude statistique}

Bruhn et al. (1982) ont essayé d'élaborer une méthode de prévision à partir d'une régression linéaire pas à pas visant à caractériser l'influence de paramètres autres que les mouvements du terrain vis-à-vis des dégradations. Cinquante individus ont été caractérisés par quatre paramètres qualitatifs et douze paramètres quantitatifs (tableau VI). Malgré des résultats statistiquement bons, cette étude ne révèle pas de paramètres fortement corrélés aux dégradations. Cependant,

TABLEAU II Classification des structures et des dégradations selon Bhattacharya et Singh (1984).

Buildings and damage classification (Bhattacharya et Singh. 1984).

Structure en briques et maçonneries, murs porteurs en briques, structure de faible hauteur 1

\begin{tabular}{|c|c|c|c|c|c|}
\hline \multicolumn{5}{|c|}{ Structure à ossature en acier ou béton armé } & 2 \\
\hline \multicolumn{5}{|c|}{ Structure à ossature bois } & 3 \\
\hline \multicolumn{5}{|c|}{ Structure massive particulièrement rigide } & 4 \\
\hline \multicolumn{6}{|c|}{ Mouvements du tertain maximaux acceptables (valeurs recommandees) } \\
\hline $\begin{array}{l}\text { Importance } \\
\text { des dégradations }\end{array}$ & $\begin{array}{l}\text { Catégorie } \\
\text { de la structure }\end{array}$ & $\begin{array}{l}\text { Angle } \\
\text { de distorsion }\left(10^{-2}\right)\end{array}$ & $\begin{array}{c}\text { Déformation } \\
\text { horizontale }\left(10^{-3}\right)\end{array}$ & $\begin{array}{c}\text { Déflexion } \\
\left(10^{-3}\right)\end{array}$ & $\begin{array}{c}\text { Rayon } \\
\text { de courbure }(\mathrm{km})\end{array}$ \\
\hline Architecturale & $\begin{array}{l}1 \\
2 \\
3 \\
4\end{array}$ & $\begin{array}{c}1 \\
1,3 \\
1,5 \\
-\end{array}$ & $\begin{array}{c}0,5 \\
- \\
-\end{array}$ & $\begin{array}{c}0,3 \\
- \\
- \\
-\end{array}$ & $\begin{array}{l}- \\
- \\
-\end{array}$ \\
\hline Fonctionnelle & $\begin{array}{l}1 \\
2 \\
3 \\
4\end{array}$ & $\begin{array}{c}2,5 \text { à } 3 \\
3,3 \\
3,3 \text { à } 5 \\
-\end{array}$ & $\begin{array}{c}1.5 \text { à } 2 \\
= \\
- \\
-\end{array}$ & $\begin{array}{c}0,5 \\
- \\
- \\
-\end{array}$ & $\begin{array}{l}20 \\
- \\
-\end{array}$ \\
\hline Structurelle & $\begin{array}{l}1 \\
2 \\
3 \\
4\end{array}$ & $\begin{array}{l}7 \\
- \\
- \\
-\end{array}$ & $\begin{array}{l}3 \\
- \\
-\end{array}$ & $\begin{array}{l}- \\
- \\
-\end{array}$ & $\begin{array}{l}- \\
- \\
-\end{array}$ \\
\hline
\end{tabular}

Critères basés sur 71 valeurs de seuil disparates issues de la bibliographie.

- Aucune valeur (manque d'information).

"L'angle de distorsion peut ètre estimé par la formule (longueur de la structure)/(2"rayon de courbure).

"* Généralement égale au tassement différentiel divisé par la longueur de l'ouvrage. 
TABLEAU III Classification des structures et des dégradations selon Yu et al. (1988).

Buildings and damage classification (Yu et al., 1988).

\begin{tabular}{|c|c|c|}
\hline 1 - Fondations & $\begin{array}{l}\text { Fondations ponctuelles } \\
\text { Fondations filantes } \\
\text { Radier } \\
\text { Buoyancy foundation }\end{array}$ & $\begin{array}{c}1 \\
4 \\
8 \\
16\end{array}$ \\
\hline 2-Matériaux de la superstructure & $\begin{array}{l}\text { Briques, pierres et béton } \\
\text { Béton armé } \\
\text { Bois } \\
\text { Acier }\end{array}$ & $\begin{array}{l}2 \\
4 \\
6 \\
8\end{array}$ \\
\hline $\begin{array}{l}3-1 / R \\
\text { "L }): \text { longueur de la structure } \\
\text { "R } n: \text { rayon de la cuvette (cas critique) }\end{array}$ & $\begin{array}{l}<0,1 \\
0,1 \text { à } 0,25 \\
0,26 \text { à } 0.5 \\
>0,5\end{array}$ & $\begin{array}{l}8 \\
6 \\
4 \\
2\end{array}$ \\
\hline $\begin{array}{l}4-\mathrm{H} / \mathrm{L} \\
\text { is } \mathrm{L} \text { : longueur de la structure } \\
\text { "Hv; hauteur de la structure }\end{array}$ & $\begin{array}{l}<1 \\
1 \text { à } 2,5 \\
2,6 \text { à } 5 \\
>5\end{array}$ & $\begin{array}{l}8 \\
6 \\
4 \\
2\end{array}$ \\
\hline
\end{tabular}

\begin{tabular}{lcccc}
\hline \multicolumn{4}{c}{ Classification de la structure } \\
\hline Nombre de points cumulés & 7 à 10 & 11 à 20 & 20 à 30 & 30 à 40 \\
\hline Classe de résistance & 1 & 2 & 3 & 4 \\
\hline
\end{tabular}

\begin{tabular}{|c|c|c|c|c|}
\hline \multicolumn{5}{|c|}{ Mouvements du terrain maximaux acceptables } \\
\hline $\begin{array}{c}\text { Importance } \\
\text { des dégradations }\end{array}$ & $\begin{array}{c}\text { Classe } \\
\text { de la structure }\end{array}$ & $\begin{array}{c}\text { Angle } \\
\text { de distorsion }\left(10^{-3}\right) \\
\end{array}$ & $\begin{array}{c}\text { Déformation } \\
\text { horizontale }\left(10^{-3}\right)\end{array}$ & Pente $\left(10^{-1}\right)$ \\
\hline Architecturale & $\begin{array}{l}1 \\
2 \\
3 \\
4\end{array}$ & $\begin{array}{c}1 \\
1,3 \\
1,5 \\
-\end{array}$ & $\begin{array}{c}0,5 \\
2 \\
3 \\
4\end{array}$ & $\begin{array}{c}2,5 \\
5 \\
10 \\
15\end{array}$ \\
\hline Fonctionnelle & $\begin{array}{l}1 \\
2 \\
3 \\
4\end{array}$ & $\begin{array}{c}2,5 \\
3,5 \\
4 \\
-\end{array}$ & $\begin{array}{c}1.5 \\
- \\
- \\
-\end{array}$ & $\begin{array}{l}- \\
- \\
- \\
-\end{array}$ \\
\hline Structurelle & $\begin{array}{l}1 \\
2 \\
3 \\
4\end{array}$ & $\begin{array}{l}5 \\
- \\
-\end{array}$ & $\begin{array}{l}3 \\
- \\
- \\
-\end{array}$ & $\begin{array}{l}- \\
- \\
-\end{array}$ \\
\hline
\end{tabular}

aucun paramètre ne prenait explicitement en compte les mouvements du terrain, ni l'aspect structural des bâtiments (état technique, rigidité...).

\section{4}

\section{Bilan sur les méthodes de prévision existantes}

L'analyse de l'ensemble des méthodes de prévision des dégradations liées aux affaissements miniers permet de réaliser un premier bilan. Un nombre important de variables et d'approches a été proposé. Cependant, certaines variables ou considérations apparaissent souvent :
- la vulnérabilité du bâti est estimée au moyen de cinq familles de critères: les caractéristiques géométriques de l'ouvrage (hauteur, longueur...), son aspect visuel extérieur, ses caractéristiques technologiques (système porteur, matériaux), le type de fondation, l'effet de site (type de terrain...) ;

- les mouvements du terrain sont caractérisés en premier lieu par la déformation horizontale, puis par l'angle de distorsion et enfin par la courbure et la pente;

- au croisement de la vulnérabilité et de l'aléa lié aux mouvements de terrain, des classes de dégradation sont définies souvent au nombre de trois ou cinq. Nous préférons n'utiliser que trois classes (architecturale, fonctionnelle et structurelle) ce qui évite une précision 
TABLEAU IV Classification des structures et des dégradations selon Dzegniuk et Hejmanowski (2000). Buildings and damage classification (Dzegniuk et Hejmanowski; 2000).

\begin{tabular}{|c|c|c|}
\hline 1 - Longueur de l'ouvrage & $\begin{array}{l}<10 \mathrm{~m} \\
11 \text { à } 15 \mathrm{~m} \\
16 \text { à } 20 \mathrm{~m} \\
21 \text { a } 25 \mathrm{~m} \\
26 \text { à } 30 \mathrm{~m} \\
31 \text { à } 35 \mathrm{~m} \\
36 \text { a } 40 \mathrm{~m} \\
>40 \mathrm{~m}\end{array}$ & $\begin{array}{l}4 \\
7 \\
11 \\
16 \\
22 \\
29 \\
37 \\
42\end{array}$ \\
\hline 2 - Forme de la structure & $\begin{array}{l}\text { Simple et compacte } \\
\text { Légèrement démembrée } \\
\text { Fortement démembrée } \\
\text { Simple et vaste } \\
\text { Démembrée et vaste }\end{array}$ & $\begin{array}{l}0 \\
3 \\
6 \\
6 \\
8\end{array}$ \\
\hline 3 -Fondations de la structure & $\begin{array}{l}\text { Sur un seul niveau, avec ou sans sous-bassement } \\
\text { Sur plusieurs niveaux } \\
\text { Sur plusieurs niveaux avec sous-bassement partielle } \\
\text { Comme précédent avec entrèe de garage }\end{array}$ & $\begin{array}{l}0 \\
3 \\
6 \\
8\end{array}$ \\
\hline 4-Terrain d'assise & $\begin{array}{l}\text { Compressible } \\
\text { Moyennement compressible } \\
\text { Incompressible }\end{array}$ & $\begin{array}{c}0 \\
4 \\
12\end{array}$ \\
\hline 5 -Type de structure & $\begin{array}{l}\text { Rigide } \\
\text { Moyennement compressible } \\
\text { Souple }\end{array}$ & $\begin{array}{l}0 \\
4 \\
8\end{array}$ \\
\hline $\begin{array}{l}6 \text { - Protections existantes } \\
\text { contre les effets miniers }\end{array}$ & $\begin{array}{l}\text { Boulonnages/ancrages } \\
\text { Partiellement boulonné/protection partielle } \\
\text { Aucune protection }\end{array}$ & $\begin{array}{r}0 \\
4 \\
12\end{array}$ \\
\hline 7 -Etat technique de la structure & $\begin{array}{l}\text { Bon } \\
\text { Moyen } \\
\text { Mauvais }\end{array}$ & $\begin{array}{r}0 \\
6 \\
12\end{array}$ \\
\hline
\end{tabular}

Classification de la structure

\begin{tabular}{lccccc}
\hline $\begin{array}{l}\text { Nombre de points } \\
\text { cumulés }\end{array}$ & $<20$ & 21 à 27 & 28 à 36 & 37 à 47 & $>48$ \\
\hline Classe de résistance & 4 & 3 & 2 & 1 & 0 \\
\hline
\end{tabular}

\begin{tabular}{cccc}
\hline $\begin{array}{c}\text { Classe } \\
\text { de rèsistance }\end{array}$ & Pente $\left(10^{-5}\right)$ & $\begin{array}{c}\text { Déformation } \\
\text { horizontale }\left(10^{-2}\right)\end{array}$ & $\begin{array}{c}\text { Rayon de courbure } \\
(\mathrm{km})\end{array}$ \\
\hline 0 & $<2,5$ & $<1,5$ & $>20$ \\
\hline 1 & 2,5 à 5 & 1,5 à 3 & 7.5 à 20 \\
\hline 2 & 5 à 10 & 3 à 6 & 4 à 7,5 \\
\hline 3 & 10 à 15 & 6 à 9 & 2,5 à 4 \\
\hline 4 & $>15$ & $>9$ & $<4$ \\
\hline
\end{tabular}

délicate à mettre en œuvre. Par ailleurs, on peut relier ces classes à des états limites : la dégradation fonctionnelle correspondant à un état limite de service, et la dégradation structurelle à un état limite ultime comme ils apparaissent dans les règlements officiels (eurocodes) :
- chacune de ces méthodes apparaît satisfaisante sur certains points et insuffisante sur d'autres. Elles révèlent souvent la difficulté d'effectuer les mesures et les observations nécessaires pour connaître l'ensemble des paramètres au niveau de chaque structure (terrain, type de structure, mouvements du terrain...). 
TABLEAUY Classification des structures et des dégradations selon Kwiatek (1998). Buildings and damage classification (Kwiatek, 1998).

\begin{tabular}{|c|c|c|c|}
\hline $\begin{array}{l}1 \text { - Dimension } \\
\text { du plan horizontal - longueur en (m) }\end{array}$ & \multicolumn{2}{|l|}{$\begin{array}{l}-\leq 10 \\
-11 \text { à } 15 \\
-16 \text { à } 20 \\
-21 \text { à } 30 \\
-31 \text { à } 40 \\
-41 \text { à } 50 \\
-51 \text { à } 60 \\
-61 \text { à } 70 \\
-71 \text { à } 80 \\
-81 \text { à } 90 \\
-\geq 91\end{array}$} & $\begin{array}{l}2 \\
4 \\
7 \\
15 \\
20 \\
25 \\
30 \\
35 \\
40 \\
45 \\
50\end{array}$ \\
\hline 2-Forme du bloc du bâtiment & \multicolumn{2}{|c|}{$\begin{array}{l}\text { - Plan simple, bloc compact } \\
\text { - Plan simple, bloc allongé } \\
\text { - Plan peu démembré, bloc compact } \\
\text { - Plan peu démembré, bloc allongé } \\
\text { - Plan fortement démembré, bloc compact } \\
\text { - Plan fortement démembré, bloc allongé }\end{array}$} & $\begin{array}{r}0 \\
2 \\
4 \\
6 \\
8 \\
10\end{array}$ \\
\hline 3-Fondation du bâtiment & \multicolumn{2}{|c|}{$\begin{array}{l}\text { - Fondation sur niveau constant } \\
\text { - Niveau de fondation variable } \\
\text { - Fondation avec porte cochère, sans cave }\end{array}$} & $\begin{array}{l}0 \\
5 \\
8\end{array}$ \\
\hline 4-Assise du bâtiment (sol) & \multicolumn{2}{|c|}{$\begin{array}{l}\text { - Sols non rocheux, sauf cailloux et enrochements } \\
\text { - Terrain remblayé } \\
\text { - Fondation sur une couche d'arnortissement } \\
\text { - Cailloux et sols rocheux, sauf roche solide ou peu fissurée }\end{array}$} & $\begin{array}{c}0 \\
4 \\
6 \\
10\end{array}$ \\
\hline \multirow[t]{5}{*}{5 - Construction du bâtiment } & $\begin{array}{l}\text { a) } \\
\text { Fondations }\end{array}$ & $\begin{array}{l}\text { - Béton armé } \\
\text { - Béton } \\
\text { - Maçonnerie en briques } \\
\text { - Pierre }\end{array}$ & $\begin{array}{l}0 \\
2 \\
3 \\
4\end{array}$ \\
\hline & $\begin{array}{l}\text { b) } \\
\text { Parois des } \\
\text { caves }\end{array}$ & $\begin{array}{l}\text { - Béton } \\
\text { - Maçonnerie en briques, } \\
\text { blocs ou blocs creux en béton } \\
\text { - Maçonnerie en pierre, błocs creux } \\
\text { en béton armé }\end{array}$ & $\begin{array}{l}0 \\
1 \\
3\end{array}$ \\
\hline & $\begin{array}{l}\text { c) } \\
\text { Plancher de } \\
\text { l'étage } \\
\text { le plus bas }\end{array}$ & $\begin{array}{l}\text { - Béton armé, Ackermann, avec couronnes } \\
\text { en béton armé } \\
\text { - Béton ou béton armé plan sur poutre en acier } \\
\text { - Plancher en segments sur poutres en acier. } \\
\text { à } U / L>1 / 10 \text { ( } \text {; largeur du segment) } \\
\text { - Plancher en segments sur poutres en acier. } \\
\text { à } / / L<1 / 10 \\
\text { - Bois avec poutres } \\
\text { - Voúte sans tirants, à } f / L>1 / 5 \\
\text { - Voúte sans tirants, à } \ell L<1 / 5\end{array}$ & $\begin{array}{l}0 \\
1 \\
2 \\
4 \\
\\
3 \\
3 \\
4 \\
8\end{array}$ \\
\hline & $\begin{array}{l}\text { d) } \\
\text { Lintatux }\end{array}$ & $\begin{array}{l}\text { - Bèton armé (monolithique ou préfabriqué) } \\
\text { ou sur poutres en acier } \\
\text { - Briques, plan } \\
\text { - Lintau en arc (arceau), à } f / L>1 / 5 \\
\text { - Lintau en arc (arceau), à } f / L<1 / 5\end{array}$ & $\begin{array}{l}0 \\
2 \\
3 \\
5\end{array}$ \\
\hline & $\begin{array}{l}\text { e) } \\
\text { Autres } \\
\text { élẻments } \\
\text { de construction }\end{array}$ & $\begin{array}{l}\text { - Arcs dans parois portantes, portée } L>1,5 \mathrm{~m} \\
\text { (sans tirants) à } f / L>1,5 \\
\text { - Arcs dans parois portantes, portée } L>1,5 \mathrm{~m} \\
\text { (sans tirants) à } f / L<1,5 \\
\text { - Hauteur de blocs de bâttiment différente } \\
\text { - Niveau de planchers différent }\end{array}$ & $\begin{array}{l}8 \\
2 \\
3\end{array}$ \\
\hline $\begin{array}{l}6 \text { - Protection contre } \\
\text { les influences minières }\end{array}$ & \multicolumn{2}{|c|}{$\begin{array}{l}\text { - Batiment protégé au niveau des fondations et tous les planchers } \\
\text { - Batiment protégé au niveau des fondations et certains planchers } \\
\text { - Bătiment protégé au niveau de tous les planchers } \\
\text { - Bătiment protégé au niveau de certains planchers } \\
\text { - Protection fragmentée } \\
\text { - Sans protection }\end{array}$} & $\begin{array}{c}0 \\
2 \\
8 \\
10 \\
12 \\
15\end{array}$ \\
\hline
\end{tabular}




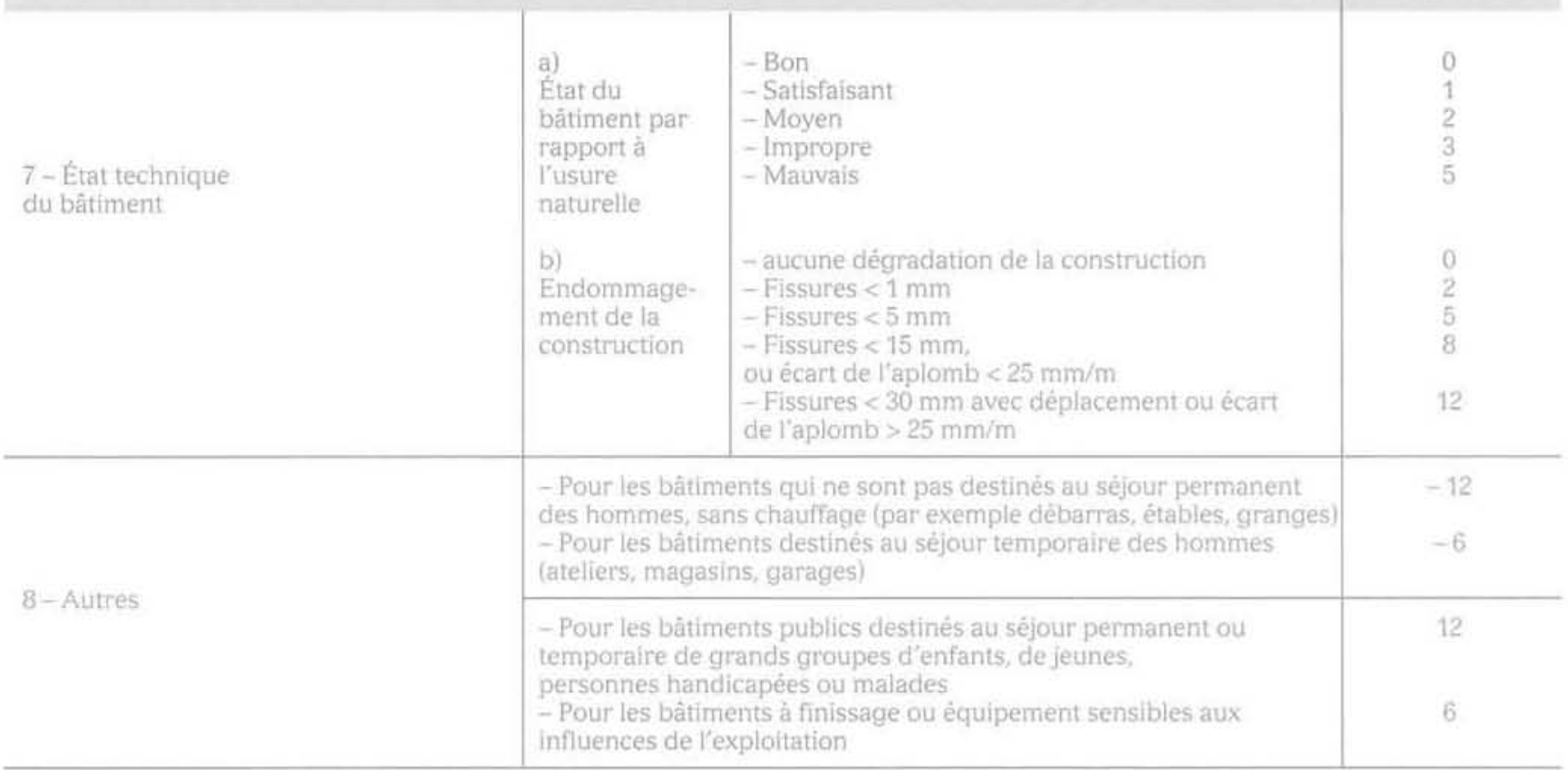

Classification de la structure et mouvements maximaux acceptables

\begin{tabular}{|c|c|c|c|c|c|c|c|c|c|c|c|}
\hline Nombre de points cumulés & $\leq 20$ & 21 à 23 & 24 à 29 & 30 à 33 & 34 à 36 & 27 à 43 & 44 à 46 & 47 à 49 & 50 à 56 & 57 à 59 & $\geq 60$ \\
\hline Classe de résistance & 4 & \multicolumn{3}{|c|}{3} & \multicolumn{3}{|c|}{2} & \multicolumn{3}{|c|}{1} & 0 \\
\hline $\begin{array}{l}\text { Déformation horizontale } \\
\text { acceptée }\left(10^{-3}\right)\end{array}$ & 9 & 6 & 5 & 4 & 3 & 2,5 & 2 & 1.5 & 1 & 0,5 & $\leq 0,3$ \\
\hline
\end{tabular}

tagleau vi Variables utilisées dans l'étude statistique de Bruhn et al. (1982).

Variables used for the statistical study of Bruhn et al. (1982).

\section{Variables qualitatives}

Origine géographique de la veine de charbon

Type d'exploitation minière en dessous

Type d'exploitation minière adjacente

Topographie autre que plate, extrémitê d'une pente ou vallée

\section{Variables quantitatives}

Surface rectangulaire au sol de la structure englobant les fondations

Ouverture de l'exploitation $\alpha$ On

Profondeur de l'expioitation $* \mathrm{H} *$

$\mathrm{O} / \mathrm{H}$

$(\mathrm{O} / \mathrm{H})^{*}$ taux de défruitement

Facteur de forme de la structure

Tan (D) avec « D o l'angle dans un plan vertical entre le bord de l'exploitation adjacente et la structure

Largeur des fondations

Taux de déflexion du mur du sous-bassement dans le plan de la longueur engendrés soit par la flexion, soit par le cisaillement

Taux de déflexion du mur du sous-bassement dans le plan de la largeur engendrés soit par la flexion, soit par le cisaillement 


\section{Étude des affaissements lorrains}

\section{Présentation}

Au cours des années 1996 à 1999, cinq affaissements miniers se sont produits dans le bassin ferrifère lorrain: rue de Metz à Auboué (1996), cité Coinville à Auboué (1996), Moutiers « Haut » (1997), Moutiers cuvette du stade (1997) et Roncourt (1999). La figure 8 présente l'exemple de la cuvette de Moutiers-Haut. Le tableau VII est une synthèse des principales caractéristiques des cinq affaissements miniers étudiés. Une base de données a été réalisée; elle regroupe le maximum d'informations sur les structures touchées par ces affaissements et les mouvements de la surface à l'origine des dégradations observées. Près de 400 habitations ont été touchées, dont 300 ont pu ètre suffisamment renseignées pour être utilisées dans une étude statistique. Les objectifs de l'étude visent à répondre aux questions fondamentales suivantes :

- Les abaques et méthodes proposés à travers le monde sont-ils vérifiés dans le bassin ferrifère lorrain?

- Certaines grandeurs, qui n'apparaissent pas dans les méthodes précédentes, peuvent être jugées pertinentes par le spécialiste en vue d'une estimation des dégradations. Est-il possible de confirmer ou d'infirmer l'intérêt de ces grandeurs?

- Est-il envisageable d'ébaucher une méthode de prévision des dégradations propre à la Lorraine, basée sur l'expérience acquise en Lorraine et à l'étranger ?

La première difficulté est de recueillir l'information nécessaire afin de tester toutes les méthodes précédentes. Il n'a pas été possible de recenser des informations précises sur l'aspect structurel même des habitations (type de fondation, rigidité, nature des différents matériaux mis en ceuvre dans l'ouvrage...) comme ce fut le cas pour un certain nombre des méthodes préalablement exposées. Beaucoup de ces constructions avaient déjà été démolies au moment de l'étude et seuls les rapports d'expertise qui avaient été établis à des fins de constat et d'indemnisation étaient disponibles. Cependant, en se référant à la classification de Bhattacharya et Singh (1984), on peut dire que la quasi-totalité des structures concernées correspond à la première catégorie (maçonnerie) et, pour certaines, à la seconde catégorie (ossature acier ou béton), On est donc susceptible d'avoir des individus suffisamment homogènes pour qu'il ne soit pas indispensable de les caractériser plus amplement du point de vue structurel. Au moyen d'une connaissance précise de l'affaissement final, il a été possible d'intégrer dans cette étude beaucoup de paramètres caractéristiques des mouvements du terrain en surface, alors que de telles données faisaient souvent défaut dans les méthodes précédentes. Au final, chaque structure a été caractérisée par quatorze variables. Le tableau I présente synthétiquement ces variables en insistant sur l'objet qu'elles caractérisent : la structure, l'affaissement ou l'interaction solstructure. Leur sens et leur détermination sont explicités ci-après. La figure 9 permet d’en préciser certaines.

Classe des dégradations «CLA » : cette variable permet de quantifier la dégradation de la structure. On a distingué trois classes, par ordre croissant de dégradation, qualifiées respectivement de " architecturale n, "fonctionnelle ) et " structurelle $\cdots$. La classe de chaque structure a été attribuée grâce à la description des dommages et à l'estimation financière des réparations (rapports d'expertise). De manière grossière, une dégradation architecturale induit un coùt inférieur à quinze mille euros et une dégradation structurelle, un coût supérieur à soixante mille euros.

Nombre d'étages \& NIV $x$ : cette variable permet indirectement de prendre en compte la hauteur de la structure. Elle n'a pu être dèterminée que pour les structures qui avaient été photographiées dans les rapports d'expertise (cuvette de Moutiers-Haut et celle du stade).

TABLEAUVII Présentation des affaissements miniers des mines de fer de Lorraine. Presentation of the French iron-ore field subidences.

\begin{tabular}{|c|c|c|c|c|c|c|c|}
\hline Site & $\begin{array}{l}\text { Nombre } \\
\text { de maisons } \\
\text { dégradées }\end{array}$ & $\begin{array}{l}\text { Profondeur } \\
\text { exploitation } \\
\text { "H } \|(\mathrm{m})\end{array}$ & $\begin{array}{l}\text { Ouverture } \\
\text { (m) }\end{array}$ & $\begin{array}{l}\text { Taux } \\
\text { défruitement } \\
\%\end{array}$ & $\begin{array}{l}\text { Affaissement } \\
\text { maximal } \\
\text { en surface } \\
\text { o. } S_{m i},(m)\end{array}$ & $\begin{array}{l}\text { Dianiètre } \\
\text { moyen de } \\
\text { la cuvelte } \\
\text { (m) }\end{array}$ & $S_{m} / H$ \\
\hline $\begin{array}{l}\text { Auboué } \\
\text { rue de Metz (1996) }\end{array}$ & 130 & 150 & 6 & 45 & 1.7 & 400 à 600 & 0,011 \\
\hline $\begin{array}{l}\text { Auboué } \\
\text { cité Coinville (1996) }\end{array}$ & 100 & 175 & 5 & 40 & 0,9 & 200 à 400 & 0,005 \\
\hline $\begin{array}{l}\text { Moutiers-Haut } \\
\text { (1997) }\end{array}$ & 70 & 120 & 3 & 55 & 1,3 & 400 à 500 & 0.011 \\
\hline $\begin{array}{l}\text { Moutiers, cuvette } \\
\text { du stade (1997) }\end{array}$ & 60 & 140 & $2,5+4$ & 55 ou 60 & $>0,55$ & 150 à 300 & $>0,004$ \\
\hline Bioncourt (1999) & 18 & 150 & 2,5 & 53 & 0,685 & 150 à 300 & 0.004 \\
\hline
\end{tabular}




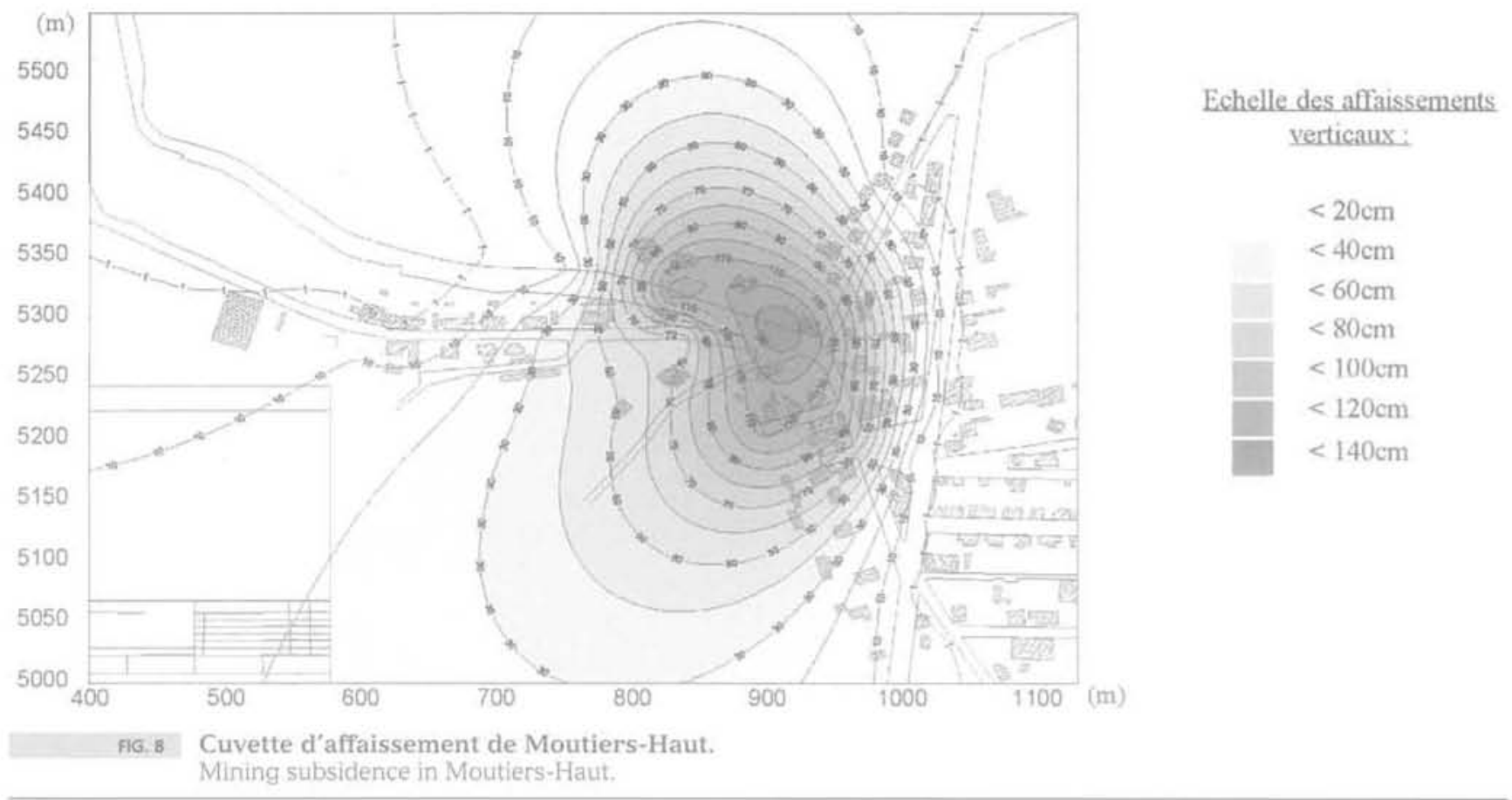

Présence d'un sous-sol « SS » : cette variable permet de prendre en compte l'existence, a priori défavorable, d'une partie enterrée de la structure. Elle a également été estimée grâce aux photographies.

Forme de la structure a FORME n : cette variable prend en compte l'aspect extérieur, compact ou démembré, de la structure. Elle a également été estimée grâce aux photographies.

Longueur de la structure « $L$ : la longueur a èté mesurée sur les plans du cadastre selon un des axes principaux de la structure, celui le plus proche de la direction de plus grande pente.

Inclinaison initiale du sol " SOLP \# : cette variable indique que la structure avait été bâtie sur un terrain incliné. Elle a été estimée grảce aux photographies et à des visites sur site.

Courbure " COURB w : au niveau de chaque structure, nous avons relevé l'affaissement le long de la direction de plus grande pente (rayon de la cuvette). La dérivée seconde de l'affaissement a permis d'estimer la courbure.

Amplitude des déformations « ADEF n: l'estimation de la déformation horizontale à la surface d'une cuvette a fait l'objet de nombreuses études empiriques pour aboutir à diverses formulations analytiques. Une description moyenne et linéarisée de ces formules a été utilisée afin de pouvoir estimer simplement l'amplitude de la déformation grâce à la seule connaissance de l'emplacement de la structure dans la cuvette (Fig. 9b).

Position de l'ouvrage dans la cuvette $\alpha$ LIEU n : cette variable caractérise la position relative de chaque structure par rapport au rayon de la cuvette d'affaissement qui passe par la structure. Elle est calculée en faisant le rapport entre la distance séparant la structure du point d'inflexion et la distance séparant le point d'inflexion du bord (respectivement du centre) de la cuvette selon que la structure est vers l'extérieur (respectivement l'intérieur) de la cuvette (Fig. 9c).
Variable «ZONE $v$ : elle permet de caractériser la zone où se situe la structure: zone de compression (entre le point d'inflexion et le centre de la cuvette) ou zone de traction (au-delà du point d'inflexion).

Variable « PENTE » : pour chaque structure, on a relevé sur le plan de la cuvette la différence d'affaissement entre ses extrémités. La pente est estimée en divisant cette différence par la longueur de l'ouvrage.

Orientation de la structure " $P / A$ » : cette variable permet de caractériser au niveau de chaque structure, l'écart angulaire dans le plan horizontal entre le grand axe de la structure et la direction de plus grande pente de la cuvette. Cette variable a pu ètre mesurée sur les plans des cuvettes superposés à ceux du cadastre (Fig. 9a).

Angle de distorsion « DIS $n$ : cette variable a été estimée grâce aux plans des cuvettes, en calculant la moitié de la différence de pente de la cuvette entre les deux extrémités de la structure. Cette démarche est parfaitement cohérente avec la définition de Boscardin et Cording (1989) sous réserve de supposer que la forme de la cuvette est localement assimilable à un cylindre. Cette variable est préférée à l'utilisation du tassement différentiel, d'emploi plus classique, mais qui ne soustrait pas le mouvement de rotation rigide de la structure qui est loin d'être négligeable lors d'un affaissement minier.

\section{2}

\section{Représentation simple des individus}

La base de données a été exploitée de plusieurs manières. Dans un premier temps, on a cherché à projeter les individus selon une représentation graphique semblable à celles du NCB (1975) et de Boscardin et Cording (1989). La figure 10 montre les résultats pour les structures localisées dans la cuvette de Moutiers, à 

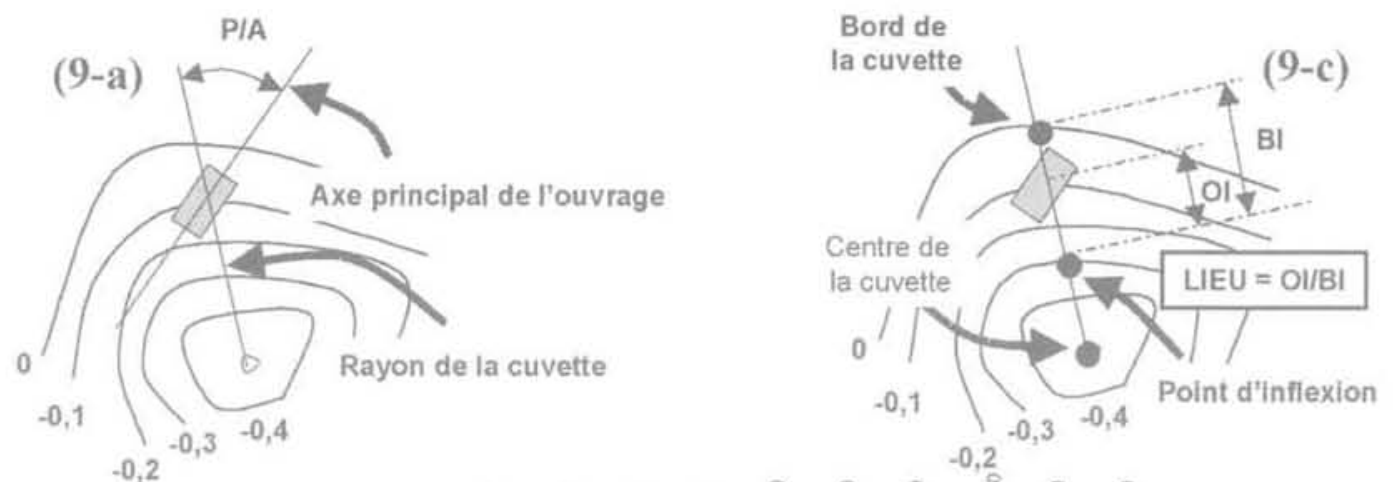

(9-b)

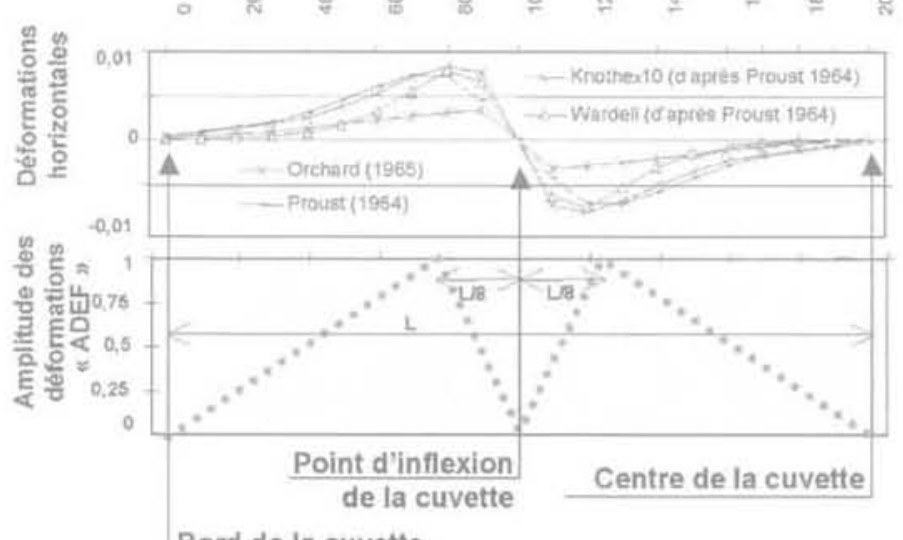

Bord de la cuvette

FIG.9 Définition des variables $« P / A »$, «LIEU $)$ et $« A D E F »$,

Definition of variables $\approx \mathrm{P} / \mathrm{A}$ », «LIEU n et $\approx A D E F$.

comparer avec ceux des figures 3 et 5 . Chaque structure est représentée selon le degré de dégradation : dégradations architecturales (cercles clairs), dégradations fonctionnelles (croix) et dégradations structurelles (carrés foncés). On observe une distribution des dégradations globalement cohérente avec celle de l'abaque du NCB (1975). Cependant, on remarque qu'il existe
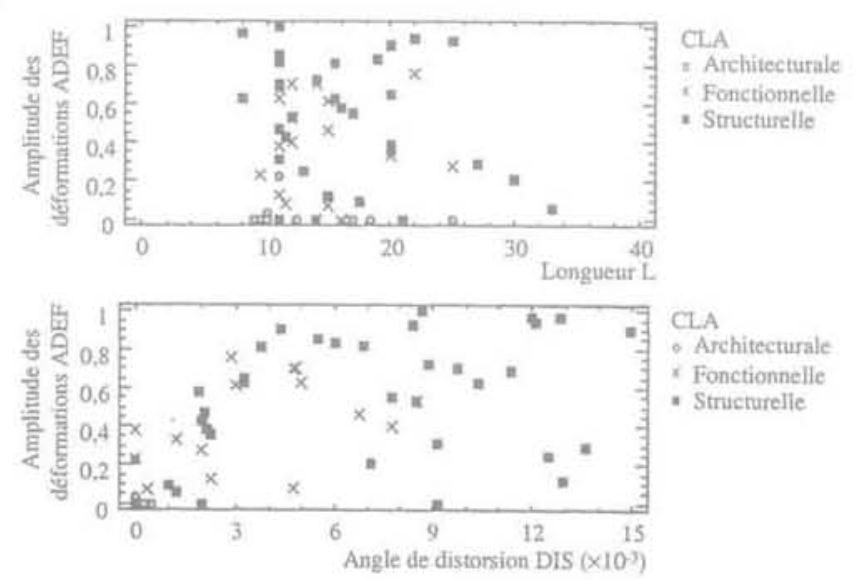

FG. 10 Répartition des dégradations observées à Moutiers selon la même représentation que l'abaque du National Coal Board (1975) et de celui de Boscardin et Cording (1989).

Similar representation of building damage in

Moutiers-Haut as the National Coal Board abacus (1975) and those of Boscardin and Cording (1989). une très grande dispersion révélatrice des limites qu'on peut accorder à cet abaque. Une observation attentive révèle par ailleurs que l'amplitude des déformations semble plus discriminante que la longueur de l'ouvrage bien que les deux variables soient pertinentes. On retrouve également la tendance de l'abaque de Boscardin et Cording (1989) malgré, là encore, une très grande dispersion. Il est probable que la distorsion est un paramètre pertinent d'un point de vue théorique mais que la difficulté pratique pour l'estimer soit rédhibitoire. En effet, il est nécessaire d'avoir une connaissance très approfondie de la morphologie d'une cuvette d'affaissement pour ètre en mesure de quantifier précisément une variation de pente entre les extrémités d'un ouvrage. De tels résultats légitiment la recherche de méthodes plus élaborées.

\section{3}

\section{Analyses multivariables}

Des études basées sur les techniques de l'analyse factorielle discriminante ont été menées dans le but de chercher des combinaisons linéaires de variables permettant la meilleure discrimination possible du type de dégradation subie par la structure. Afin d'écarter l'effet de site, on a étudié chaque cuvette séparément, puis elles ont été comparées entre elles. Les résultats des analyses factorielles discriminantes réalisées sont présentés en deux ètapes :

- analyse des seuls individus de la cuvette de MoutiersHaut. Ces derniers sont, en effet, les seuls pour lesquels toutes les variables présentées dans le tableau VIII ont 
TABLEAU VII Présentation des variables utilisées pour l'étude statistique des affaissements miniers lorrains, Presentation of variables used for the statistical study.

\begin{tabular}{|c|c|c|c|}
\hline & Variables & Signification de la variable & Amplitude de variation \\
\hline \multirow{5}{*}{ 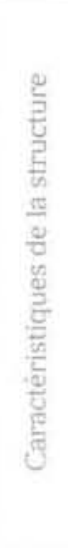 } & CLA & Classe de dégradation & $\begin{array}{l}1 \text { : architecturale } \\
2 \text { : fonctionnelle } \\
3 \text { : structurelle }\end{array}$ \\
\hline & NIV & $\begin{array}{l}\text { Nombre d'étages en plus du rez-de-chaussêe } \\
\text { (grenier compris) }\end{array}$ & 0 à 3 \\
\hline & SS & Nombre de sous-sol & $\begin{array}{l}0 \text { : aucun } \\
0,5 \text { : semi-enterré } \\
1 \text { : complètement enterré }\end{array}$ \\
\hline & FORME & $\begin{array}{l}\text { Indicateur de la forme plus ou moins complexe } \\
\text { et donc préjudiciable de l'ouvrage }\end{array}$ & $\begin{array}{l}\text { 1: forme homogène non préjudiciable } \\
2 \text { : forme moyennement complexe et } \\
\text { préjudiciable } \\
3 \text {; forme complexe et préjudiciable }\end{array}$ \\
\hline & L & Longueur de la structure & 8 a $33 \mathrm{~m}$ \\
\hline \multirow{6}{*}{  } & SOLP & Sol en pente initialement & $0:$ non, $1:$ oul \\
\hline & COURB & Courbure du terrain au niveau de l'ouvrage & 0 aे $2.10^{-3}$ correspond à un rayon $<500 \mathrm{~m}$ \\
\hline & ADEF & $\begin{array}{l}\text { Amplitude des déformations horizontales } \\
\text { du terrain au niveau de l'ouvrage }\end{array}$ & $\begin{array}{l}0 \text { : déformation nulle } \\
\text { 1: déformation maximale (traction ou } \\
\text { compression) }\end{array}$ \\
\hline & LIEU & $\begin{array}{l}\text { Position de l'ouvrage le long d'un rayon } \\
\text { de la cuvette relativement au point } \\
\text { d'inflexion }\end{array}$ & $\begin{array}{l}0 \text { : ouvrage au niveau du point d'inflexion } \\
0<\text { lieu }<1 \text { : ouvrage entre le point d'inflexion } \\
\text { et le bord ou le centre de la cuvette } \\
\text { lieu > ouvrage à l'extérieur de la cuvette }\end{array}$ \\
\hline & ZONE & $\begin{array}{l}\text { Zone de déformation horizontale dans laquelle } \\
\text { se trouve la structure }\end{array}$ & $\begin{array}{l}1: \text { zone de traction (vers l'extérieur de la } \\
\text { cuvette) } \\
-1: \text { zone de compression (vers l'extérieur de } \\
\text { la cuvette) }\end{array}$ \\
\hline & PENTE & Pente du terrain au niveau de l'ouvrage & 0 à $6 \%$ \\
\hline \multirow{2}{*}{ 돌 } & $\mathrm{P} / \mathrm{A}$ & Orientation de la structure & 0 à 45 degrés \\
\hline & Dis & Angle de distorsion au niveau de l'ouvrage & 0 à $3,5.10^{-1}$ \\
\hline
\end{tabular}

été renseignées, en particulier les variables «NIV »,

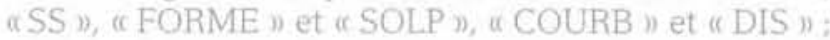
- analyse de toutes les cuvettes avec un nombre restreint de variables, en l'occurrence celles qui semblent les plus pertinentes au vu de l'étude précédente et/ou celles qui sont renseignées pour les autres cuvettes.

Les résultats des analyses sont présentés par l'intermédiaire de trois composantes:

- une figure représentant la projection des individus dans un plan défini par les deux axes discriminants : - un tableau décrivant chacun des deux axes discriminants par l'intermédiaire de coefficients de fonctions discriminantes. Le poids d'une variable est d'autant plus important que le coefficient attribué est élevé. Par ailleurs, le signe du coefficient renseigne sur le sens de la corrélation qui peut exister entre chaque variable et la discrimination des dégradations :

- un tableau représentant les résultats de l'opération d'attribution d'une classe de dégradation à chaque individu à l'aide des fonctions discriminantes, en fajsant évidemment abstraction de leur classe réelle. En comparant la classe réelle à la classe attribuée par la méthode, on peut ainsi tester son efficacité.
L'analyse du cas de Moutiers-Haut (Fig. 11) met en évidence la possibilité de discriminer les dégradations. On observe un bon résultat du reclassement $(27,5 \%$ d'erreur) compte tenu de l'imprécision qui entache la détermination de chaque variable. L'axe horizontal discrimine les groupes de manière satisfaisante puisque la dégradation décroit régulièrement le long de ce dernier. Par ailleurs, on observe avec satisfaction que les individus mal reclassés sont en grande majorité affectés à un groupe de dégradation directement adjacent à leur groupe d'origine. L'analyse met en évidence l'importance primordiale des mouvements de la surface. Les variables "PENTE », "ZONE», "ADEF ») et "L.IEU $\gg$ sont celles qui ont le plus de poids dans la définition des fonctions discriminantes. L'importance du coefficient attribué à la variable « LIEU » est intéressante, car cette variable est très simple à estimer et donc à utiliser. On observe, en revanche, une participation très modeste des paramètres tels que l'angle de distorsion de la structure "DIS » ou la courbure " COURB $x$ par rapport aux précédentes variables. En réalité, la courbure et l'angle de distorsion sont calculés à partir de la variation de la pente le long de l'ouvrage. Ce sont donc des dérivées secondes de l'affaissement vertical, ce qui nécessite une excellente connaissance 

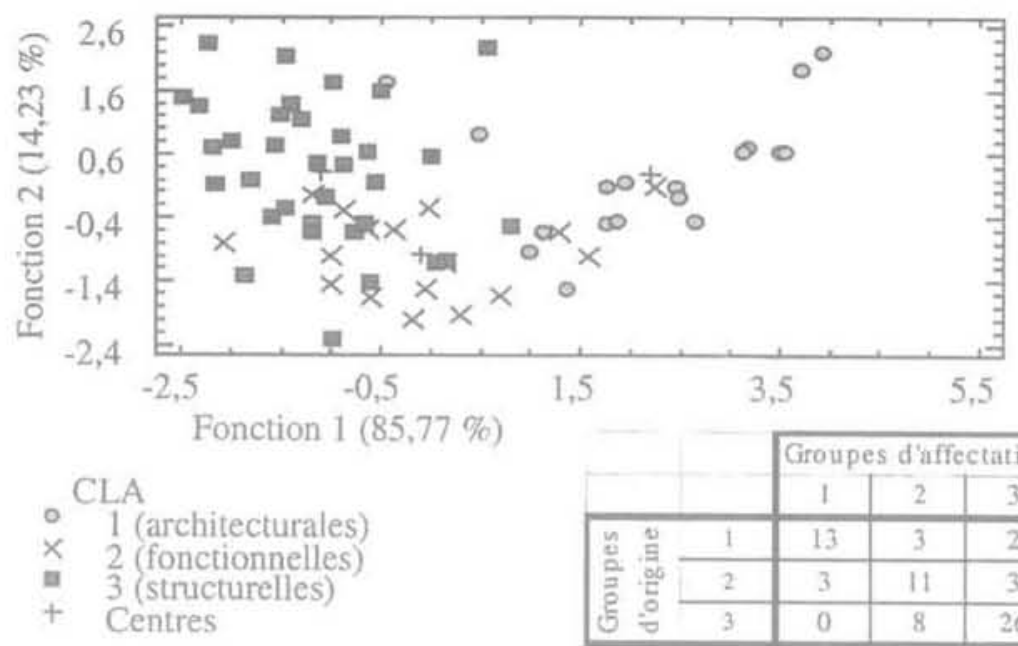

Fonctions discriminantes standardisées

\begin{tabular}{|c|c|c|}
\cline { 2 - 3 } \multicolumn{1}{c|}{} & Fonction 1 & Fonction 2 \\
\hline ADEF & $-0,33$ & 0,24 \\
\hline COURB & $-0,05$ & $-0,15$ \\
\hline DIS & $-0,14$ & $-0,03$ \\
\hline FORM & 0,03 & 0,11 \\
\hline L & $-0,21$ & 0,40 \\
\hline LIEU & 0,63 & 0,70 \\
\hline NIV & 0,03 & 0,30 \\
\hline P/A & $-0,04$ & $-0,21$ \\
\hline PENTE & $-0,22$ & 1,16 \\
\hline SOLP & $-0,35$ & $-0,16$ \\
\hline SS & $-0,02$ & $-0,39$ \\
\hline ZONE & $-0,15$ & 0,59 \\
\hline
\end{tabular}

FIG.11 Résultats de l'analyse factorielle discriminante avec les individus de la cuvette de Moutiers-Haut. Discriminant analysis with observations in Moutiers-Haut.

de l'affaissement pour qu'eux-mêmes soient significatifs. Ceci n'est pas le cas de la déformation horizontale qui est estimée à partir d'une variation théorique le long de la cuvette. Il est évident que cette méthode donne des résultats beaucoup moins sensibles aux imprécisions de la cuvette. Par ailleurs, on sait que la courbe de variation de la déformation horizontale et celle de la courbure sont quasi isomorphes (Fig. 1). Dans le cadre d'une étude statistique, et même si la courbure et la déformation sont deux phénomènes physiques différents, la courbure se trouve d'une certaine manière prise en compte par la variable déformation «ADEF ». Ces deux raisons peuvent expliquer le faible poids de la courbure dans la discrimination. On peut conclure de cette étude qu'il est satisfaisant de ne conserver que les quatre variables ( ADEF ), "LIEU ), "ZONE » et « PENTE » pour caractériser les mouvements de la surface. Ce sont ces dernières qui apparaitront dans l'analyse des individus des autres cuvettes.

Dans une seconde étape, nous avons souhaité comparer les résultats des analyses discriminantes effectuées sur les cinq cuvettes d'affaissement. La comparaison se fait essentiellement sur les variables communes aux cinq études. En effet, certaines variables ont montré qu'elles n'étaient pas pertinentes compte tenu des autres variables (c'est le cas des variables $\approx$ COURB $»$ et $«$ DIS $»)$, ou ont été mal rensejgnées (c'est le cas de $\alpha$ NIV $), ~ «$ SS $)$ et $~($ FORME $»)$. Les résultats de ces analyses sont présentés sur la figure 12. Pour la cuvette du Stade, seulement deux classes sont à discriminer, ce qui explique qu'il n'y ait qu'une seule fonction discriminante. Une première observation montre que la discrimination est correcte pour toutes les cuvettes, sauf pour celle d'Auboué, rue de Metz. Par ailleurs, on constate que l'analyse de la cuvette de Moutiers-Haut, réalisée cette fois avec moins de variables que précédemment, aboutit à des résultats très similaires, ce qui justifie a posteriori le choix d'exclure certaines variables peu discriminantes.

Il reste alors à exploiter les résultats des analyses en vue de proposer une hiérarchisation des facteurs déterminants pour l'estimation des dégradations, qu'on comparera ensuite aux diverses méthodes présentées. Pour ce faire, on a décidé de faire une « moyenne » de l'influence de chaque variable. Afin de ne pas s'encombrer d'une précision inutile, on a noté pour chaque axe l'importance relative des variables les unes par rapport aux autres. Cette opération revient, en quelque sorte, à normer chaque fonction discriminante afin de pouvoir les comparer entre elles. Nous avons distingué quatre quarts pour l'axe le plus discriminant et deux moitiés pour l'axe le moins discriminant. En fonction du quart (respectivement de la moitié) d'appartenance de chaque variable, une valeur de 1 à 4 (respectivement de 1 ou 2) a été attribuée à la variable. La valeur minimale indique une part mineure de la variable dans la fonction discriminante alors que la valeur maximale indique une participation majoritaire. Cette distinction entre les deux axes s'explique par le pourcentage de l'inertie attribué à chacun d'eux qui est de l'ordre de $80 \%$ pour le premier et $20 \%$ pour le second. Pour chaque analyse, la note finale attribuée à une variable est la valeur maximale entre le numéro de quartile et celui de la moitié, Par ailleurs, un signe positif de la note signifie qu'une augmentation de la variable tend à augmenter la dégradation du bâti et inversement pour un signe négatif.

Le tableau IX récapitule les résultats obtenus. Les lignes correspondant aux variables « P/A » et « ZONE $»$ apparaissent en blanc signifiant que l'influence de ces variables vis-à-vis des dégradations n'est pas la même pour tous les cas ; elle peut être successivement favorable puis défavorable. Il semble donc délicat d'en tenir compte. Toutes les autres variables (lignes grisées) agissent conformément à nos connaissances, mais surtout globalement dans le même sens pour toutes les analyses. La liste des critères pertinents dans l'ordre décroissant de leur pouvoir discriminant est donc la suivante : "LIEU », "L », "ADEF », " PENTE », “FORME », "SS ». La variable « ADEF », qui caractérise la déformation horizontale, ne sort pas en tête de ce classement. C'est en effet la variable « LIEU n, caractérisant la position relative de la structure par rapport au point d'inflexion, qui est la plus discriminante. Or d'un point de vue pratique, la déformation horizontale est plus délicate à estimer que la variable « LIEU », Qui plus est, cette dernière a une signification proche de celle de la déformation horizontale puisqu'elle prend 


\section{Cuvette de Moutiers Haut :}

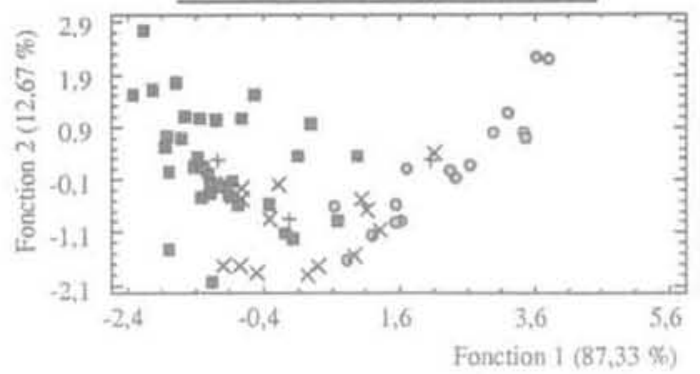

\begin{tabular}{|c|c|c|}
\cline { 2 - 3 } \multicolumn{1}{c|}{} & Fonction 1 & Fonction 2 \\
\hline ADEF & $-0,36$ & 0,23 \\
\hline L & $-0,27$ & 0,46 \\
\hline LIEU & 0,67 & 0,74 \\
\hline P/A & $-0,10$ & $-0,33$ \\
\hline PEN TE & $-0,22$ & 1,00 \\
\hline ZONE & $-0,11$ & 0,61 \\
\hline
\end{tabular}

\begin{tabular}{|c|c|c|c|c|}
\hline & & \multicolumn{3}{|c|}{ Groupes d'affectation } \\
\hline & & 1 & 2 & 3 \\
\hline \multirow{3}{*}{ 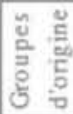 } & 1 & 13 & 3 & 2 \\
\hline & 2 & 1 & 12 & 4 \\
\hline & 3 & 1 & 7 & 26 \\
\hline
\end{tabular}

Cuvette du Stade à Moutiers:

\begin{tabular}{|c|c|}
\cline { 2 - 2 } \multicolumn{1}{c|}{} & Fonction I \\
\hline ADEF & -0.03 \\
\hline FORME & -0.59 \\
\hline L & $-0,38$ \\
\hline LIEU & 0.92 \\
\hline NIV & 0.11 \\
\hline P/A & 0,03 \\
\hline SS & -0.41 \\
\hline
\end{tabular}

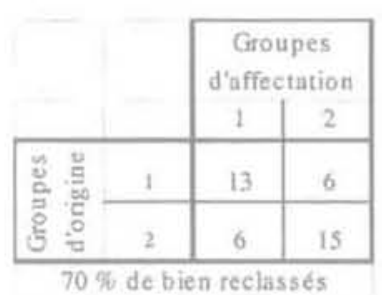

Cuvette de la rue de Metz:



\begin{tabular}{|c|c|c|}
\cline { 2 - 3 } \multicolumn{1}{c|}{} & Fonction 1 & Fonction 2 \\
\hline A DEF & 0,04 & -0.39 \\
\hline L & $-0,37$ & -0.31 \\
\hline L.BU & 0.01 & -0.22 \\
\hline P/A & 1.05 & -0.02 \\
\hline PENTE & $-0,26$ & 0,76 \\
\hline ZONE & 0.21 & 0,93 \\
\hline
\end{tabular}

\begin{tabular}{|c|c|c|c|c|}
\hline & & \multicolumn{3}{|c|}{ Groupes d'affectation } \\
\hline & & 1 & 2 & 3 \\
\hline \multirow{3}{*}{ 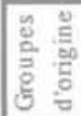 } & 1 & 22 & 6 & 3 \\
\hline & 2 & 7 & 8 & 5 \\
\hline & 3 & 5 & 7 & 23 \\
\hline
\end{tabular}

Cuvette de la cité Coinville:

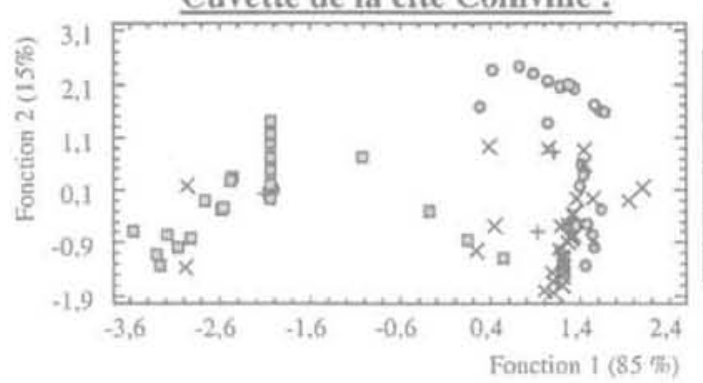

\begin{tabular}{|c|c|c|}
\cline { 2 - 3 } \multicolumn{1}{c|}{} & Fonction I & Fonction 2 \\
\hline ADEF & $-0,26$ & $-0,64$ \\
\hline L & $-0,43$ & 0,36 \\
\hline LIEU & $-0,42$ & 0,49 \\
\hline P/A & 0,00 & $-0,02$ \\
\hline PEN TE & $-0,54$ & 0,09 \\
\hline ZONE & 1,14 & $-0,11$ \\
\hline
\end{tabular}
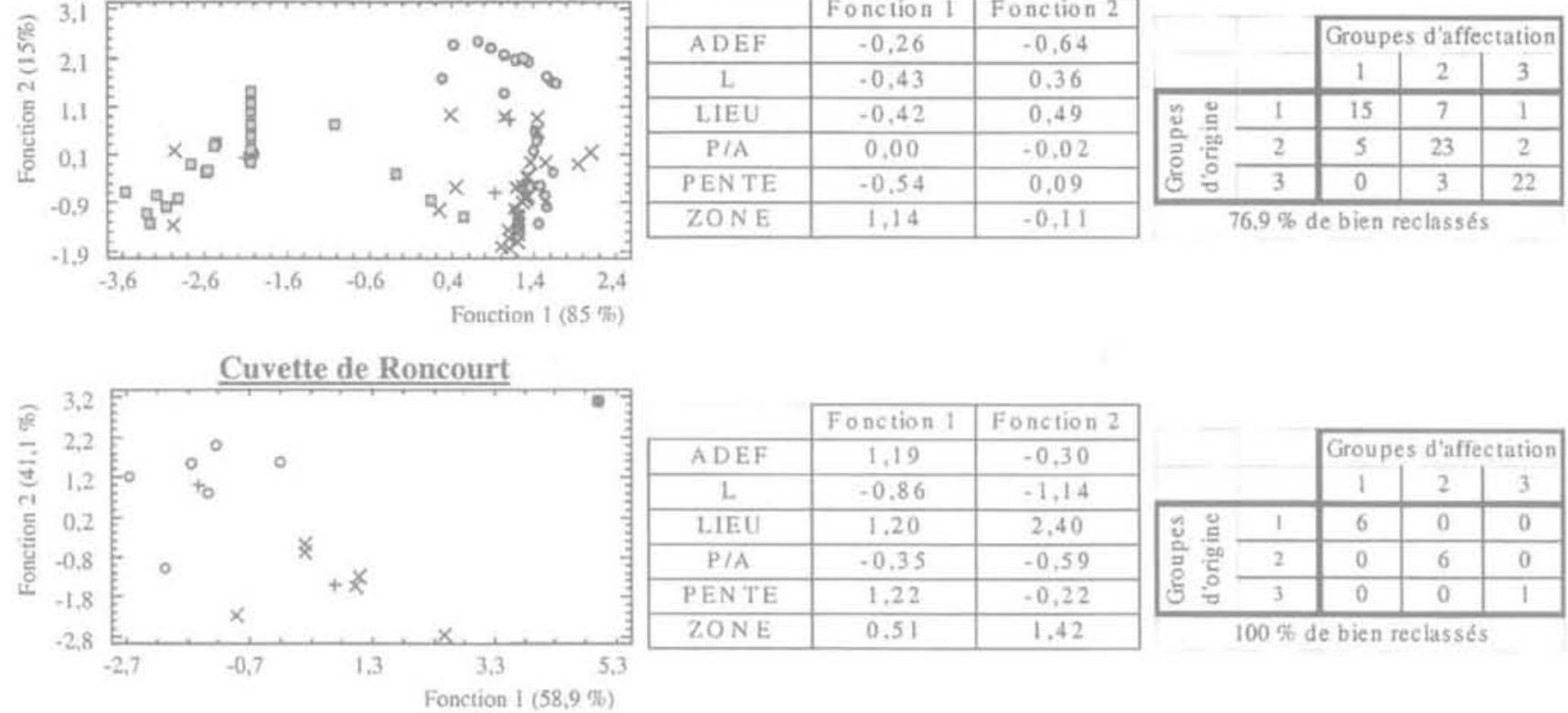

\begin{tabular}{|c|c|c|}
\cline { 2 - 3 } \multicolumn{1}{c|}{} & Fonction 1 & Fonction 2 \\
\hline ADEF & 1,19 & $-0,30$ \\
\hline L & -0.86 & $-1,14$ \\
\hline L.IEU & 1.20 & 2,40 \\
\hline P/A & -0.35 & $-0,59$ \\
\hline PENTE & 1,22 & $-0,22$ \\
\hline ZONE & 0.51 & 1.42 \\
\hline
\end{tabular}

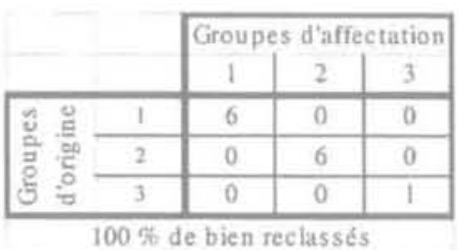

FiG. 12 Résultats des analyses factorielles discriminantes avec les individus des cinq cuvettes. Discriminant analysis with observation of the five subsidences: 
TABLEAUIX Synthèse de l'importance de chaque variable pour la prévision des dégradations. Synthesis of criteria weight for mining damage prediction.

\begin{tabular}{|c|c|c|c|c|c|c|}
\hline Variable & Moutiers-Haut & $\begin{array}{c}\text { Stade } \\
\text { (Moutiers) }\end{array}$ & $\begin{array}{l}\text { Cité Coinville } \\
\text { (Auboué) }\end{array}$ & $\begin{array}{l}\text { Rue de Metz } \\
\text { (Auboué) }\end{array}$ & Roncourt & Moyenne \\
\hline NTV & 0 & 0 & - & - & - & 0 \\
\hline SS & 0 & 2 & - & - & - & 1 \\
\hline FORME & 0 & 3 & - & - & - & 1,5 \\
\hline SOLP & 3 & - & - & - & - & - \\
\hline $\mathrm{P} / \mathrm{A}$ & 0 & 1 & 0 & -4 & 0 & - \\
\hline L & 2 & 3 & 1 & 2 & 1 & 2 \\
\hline $\mathrm{ADEF}$ & 3 & 0 & 1 & 0 & 3 & 1,5 \\
\hline LIEU & -4 & -4 & 0 & 0. & -2 & 2 \\
\hline ZONE & 1 & - & -4 & -1 & -1 & -1 \\
\hline PENTE & 1 & - & 2 & 2 & 3 & 1.5 \\
\hline
\end{tabular}

Poìds des variables $; 0 \rightarrow$ aucun, $1 \rightarrow$ faible, $2 \rightarrow$ moyen. $3 \rightarrow$ important, $4 \rightarrow$ prépondérant, $\rightarrow$ indėterminè

des valeurs défavorables aux alentours du point d'inflexion, c'est-à-dire très proche des zones de déformation maximale et/ou d'inclinaison maximale. D'une certaine manière, cette variable inclut donc la déformation horizontale et la pente. Cependant, la connaissance précise de la déformation n'en reste pas moins intéressante. On rappelle en effet que l'allure de la variation des déformations le long de la cuvette est théoriquement identique à celle de la courbure et que c'est finalement ce paramètre qui est principalement pris en compte par la variable « ADEF » de l'étude statistique.

Par ailleurs, il semble acquis que l'augmentation de la longueur de la structure est préjudiciable. La forme d'ensemble et la présence d'un sous-sol également sans que ce soit rédhibitoire. Mais comme ces résultats ne sont basès que sur deux études, il convient d'être prudent dans les conclusions. C'est là le signe que, malgré le caractère néfaste de certains aspects architecturaux, il est possible de les inclure dans une construction à condition que la conception d'ensemble de l'ouvrage en tienne compte.

Cependant, on voit que les résultats obtenus par l'étude statistique sont perfectibles. On n'a d'ailleurs pas pris en compte les aspects structuraux des ouvrages (fondations et superstructure). Ce dernier point est aisément perfectible pour la prévision des dégradations des ouvrages d'un site présentant un risque d'affaissement puisque, dans ce cas, les structures seront visitables.

\section{4}

\section{Analyse et synthèse des résultats}

Afin de comparer l'ensemble des méthodes de prévision des dégradations des structures situées en zone minière, on a repris les distinctions entre critères, proposées dans différentes méthodes, à savoir : les critères caractérisant la vulnérabilité du bâti et ceux caractéri- sant l'aléa, c'est-à-dire les mouvements de la surface induits par des instabilités souterraines, Les critères de vulnérabilité sont classés en cinq familles et les critères d'aléa sont au nombre de quatre (tableau X). Chaque colonne synthétise une méthode pour laquelle on a estimé grossièrement le poids relatif, en pourcentage, de chaque famille ou critère dans la méthode. La dernière colonne correspond aux résultats de cette étude. Il apparait, alors, dans quelle proportion chaque famille de critère doit être considérée lors de la prévision. Cette étude souffre néanmoins du manque d'informations concernant les caractéristiques structurelles du bâti. Mais grâce aux autres méthodes, on peut estimer entre 40 et $60 \%$ le poids de cet aspect (fondations et superstructure). Cette étude, pondérée par les méthodes existantes, permet d'estimer le poids des autres critères, à savoir environ $10 \%$ pour l'aspect extérieur, $30 \%$ pour les dimensions de la structure et $10 \%$ pour l'effet de site. Cependant, on peut penser que l'effet de site, en particulier les caractéristiques du terrain, est sous-estimé. Il suffit pour s'en persuader de se référer aux travaux de Peng et Cheng (1981) qui ont constaté l'efficacité du creusement de tranchées, remplies ensuite de matériaux très déformables, autour des structures. La modification du comportement du terrain qui en résulte permet de minimiser fortement les dégradations.

La caractérisation de l'affaissement devra se faire par l'intermédiaire de la variable « LIEU » qui caractérise la position relative de la structure dans la cuvette d'affaissement par rapport au point d'inflexion. On a vu que cette variable était simple d'utilisation et fortement corrélée aux dégradations. L'estimation de la pente est le second facteur. Notons à cet égard que l'inclinaison est en réalité plus responsable d'une perte de fonctionnalité de la structure que d'une dégradation structurelle. Enfin, la courbure et l'angle de distorsion peuvent apporter un complément de hiérarchisation, mais il faudra pour cela avoir une connaissance exceptionnelle de la cuvette d'affaissement. Par ailleurs, il ne semble 
TABLEAUX Synthèse des critères adoptés pour la hiérarchiation et poids respectif $(\%)$. Synthesis of criteria used for mining damage prediction.

\begin{tabular}{|c|c|c|c|c|c|c|c|c|}
\hline & $\begin{array}{l}\text { NCB } \\
11975) \\
\text { Fig.2 }\end{array}$ & $\begin{array}{l}\text { Boscandin } \\
\text { et Cording } \\
\text { ilsag) } \\
\text { Fig. } 4\end{array}$ & $\begin{array}{l}\text { Wagner } \\
\text { Stuumann } \\
\text { (12984) } \\
\text { Eig. } 5\end{array}$ & $\begin{array}{l}\text { Bhiattacharya } \\
\text { et Singh } \\
\text { (1954) } \\
\text { Tab. II }\end{array}$ & $\begin{array}{l}\text { Yu } \\
\text { etal. } \\
\text { [1988] } \\
\text { Tah. III }\end{array}$ & $\begin{array}{l}\text { Dhegniuk } \\
\text { et } \\
\text { Hejmanowski } \\
(2000)\end{array}$ & $\begin{array}{l}\text { Kwiatek } \\
\text { [15S6) } \\
\text { Tab. V } \\
\text { Tab. IV }\end{array}$ & $\begin{array}{l}\text { Affaissements } \\
\text { lorrains } \\
\text { Tab, IX }\end{array}$ \\
\hline \multicolumn{9}{|c|}{ Critères visant à caractériser la vulnérabilité } \\
\hline Aspect extérieur (forme) & & & & & & 10 & 10 & 25 \\
\hline $\begin{array}{l}\text { Caractéristiques technologiques } \\
\text { de l'ouvrage (système portewr, } \\
\text { état technique, protections, } \\
\text { matériaux...) }\end{array}$ & & & & 100 & 20 & 30 & 40 & $?$ \\
\hline $\begin{array}{l}\text { Dimensions de la structure } \\
\text { (longueur, hauteur) }\end{array}$ & 100 & 100 & 100 & & 40 & 40 & 30 & 75 \\
\hline Effet cle site (terrain, géométrie...) & & & & & & 10 & 10 & ? \\
\hline Type de fondations, sous-sol & & & & & 40 & 10 & 10 & ? \\
\hline \multicolumn{9}{|c|}{ Critères visant à caractériser l'aléa } \\
\hline Déformation horizontale & 100 & 50 & 65 & 50 & 40 & 33 & 100 & 33 \\
\hline Ditorsion ou courbure & & 50 & & 50 & 40 & 33 & & 0 \\
\hline Pente & & & 35 & & 20 & 33 & & 33 \\
\hline Lieu & & & & & & & & 33 \\
\hline
\end{tabular}

pas sérieux de vouloir quantifier rigoureusement la courbure et la déformation horizontale du terrain. On a vu que la méthode proposée ici, par l'intermédiaire de la variable « $A D E F$ », semble amplement suffisante pour les caractériser toutes les deux. Notons, toutefois, que si la cuvette d'affaissement n'est pas fixe, ce qui peut être le cas lorsque la zone d'exploitation effondrée se déplace, ce sont les valeurs maximales des variables en un point qui deviennent pertinentes.

Si l'on devait préconiser ou élaborer une méthode, le choix se porterait plutôt sur une démarche qui permet une classification simple des structures prenant en compte, par exemple, le type de fondation, le type de superstructure, la forme d'ensemble, les caractéristiques du terrain et la longueur de la structure. Cette première approche ne permet qu'une discrimination grossière de la vulnérabilité. Il est évident que chaque structure doit être expertisée et on ne peut que suggérer de s'inspirer des méthodes décrites ici, en particulier des méthodes polonaises qui sont les plus complètes, et qui mettent clairement en évidence les aspects singuliers d'un ouvrage, susceptibles d'en augmenter sa vulnérabilité.

Concernant la définition des seuils permettant de caractériser le risque de dégradation pour les mouvements du terrain escomptés, il est possible d'en déterminer des valeurs sur la base de connaissances scientifiques telles que celles présentées dans le tableau II. Cependant, on ne saurait exclure de cette étape la prise en compte de considérations sociales, économiques et politiques qui ne sont pas du seul ressort du scientifique. D'autres études sont en cours actuellement, dans le cadre du Groupement de recherche sur l'impact et la sécurité des ouvrages souterrains (GISOS), pour la prise en compte de ces aspects.

\section{5}

\section{Conclusion}

L'objectif de l'étude était de comparer les méthodes de prévisions des dégradations affectant le bâti soumis à un affaissement minier afin d'élaborer une méthode de prévision propre au bassin ferrifère lorrain. Les principales conclusions formulées à partir de cette étude sont les suivantes :

- les principaux critères permettant de caractériser la vulnérabilité du bâti sont la longueur, la typologie du bảti, la forme architecturale plus ou moins complexe et le type de fondations. L'étude statistique réalisèe avec les affaissements miniers lorrains, a confirmé l'importance de la longueur et de la forme du bâti ;

- les principaux critères permettant de caractériser l'aléa sont la déformation horizontale des terrains, la pente et la courbure. L'étude statistique réalisée avec les affaissements miniers lorrains a confirmé l'importance de la déformation horizontale et de la pente :

- l'étude statistique a révélé la difficulté d'estimer et donc d'utiliser la courbure du terrain ou la distorsion de la structure pour quantifier l'aléa. En revanche, la variable LIEU, caractérisant simplement la position de la structure par rapport au point d'inflexion de la cuvette d'affaissement s'est révélée particulièrement pertinente et simple d'utilisation; 
- cette analyse permet d'approfondir les modèles de prévision des dégradations. Son application à un site à risque permettra d'améliorer son efficacité.

\section{REMERCIEMENTS}

Les travaux de recherches ont été réalisés graice aux subventions du ministère de l'Aménagement du Territoire et de l'Environnement du ministère de l'Economie, des Finances et de l'Industrie. de celui de la Recherche ainsi que des programmes INTERREG L et RESIDER de IUnion européenne dans le cadre du Groupement de recherche sur l'impact et la sécurité des ouvrages souterrains (GISOS). La région Lorraine est remerciée pour le financement octroyé dans le cadre du Contrat de Plan Etat-Région ainsi que pour le financement de thèses.

\section{Bibliographie}

Bell F.G. - $\pi$ Ground movements due to subsidence and theit influence on construction $x$. Geomechanics and water enqineering in environnemental management. 1992, p. 325-347.

Bhattacharya S., Singh M.M. - $\alpha$ Proposed criteria for subsidence damage to buildings $\mathrm{v}$. Rock mechanics in productivity and protection, 25th Symposium on rock mechanics, 1984, p. 747-755.

Boscardin M.D., Corcing E.J. - « Building response to excavationinduced settlement x. Journal of Geotechnical Engineering, vol. 115, $n^{\circ} 1,1989$

Bruhn R.W., McCann W.S., Speck R.C., Gray R.E. - « Darnage to structures above active underground coal mines in the nor- thern appalachian coal field $\%$. First Int. Conf, on stability in underground mining, chapitre 47. 1982, p. 1022-1046.

Dzegniuk B.. Hejmanowski R, - a Evaluation of the damage hazard to building objects on the mining areas considering the deformation course in time n. International congress of the international society for mine surveying, septembre 2000, CraCovie, Pologne.

Geddes J.D. - Structural design and ground movements. Ground movement and their effects on structures. Surrey University Press, 1984.

Kwiatek J. - Protection des constructions sur les terrains miniers (traduction du polonais, titre original : Ochrona obiektow budowlanych na terenach gorniczych n), publication du GIG, Katowice, 1998.

National Coal Board - Subsidence engineer's handbook 1975.

Orchard R.J., Allen W.S. - a Ground curvature due to coal mining n. Chartered surveyor, vol. 97,1965, p. 622-631.

Peng S.S. Cheng S.L. - * Predicting surface subsidence for damage prevention 13, Coal Mining Processing, vol. 18, $n^{2} 5$, mai 1981. p. 84-85.

Piguet J.-P., Wojtkowiak F, - « Affaissements et déformations audessus des exploitations minières : mécanismes et évolution dans le temps n. Les techniques de l'industrie minérale, $n^{2} 10$. uin 2001, p. 5-16,

Proust A. - Etude sur les affaissements miniers dans le bassin du Nord-Pas-de-Calais, 1964

Wagner H., Shümann E.H.R. - * Surface effects of total coal-seam extraction by underground mining methods $n$. Journal of the South African institute of mining and metallurgy, vol. $91, \mathrm{n}^{\circ} 7$. juillet 1991, p. 221-231.

Yu Z., Karmis M., Jarosz A., Haycocks C. - Development of damage criteria for buildings affected by mining subsidence. 6 th annual workshop generic mineral technology center, mine system design and ground control, juillet 1988, p. 83-92. 